\title{
Balanced truncation of linear time-invariant systems over finite-frequency ranges
}

\author{
Peter Benner ${ }^{1,2}$ (D) $\cdot$ Xin Du ${ }^{3}$. Guanghong Yang ${ }^{4}$ Dan Ye \\ Received: 1 October 2018 / Accepted: 27 October 2020 / \\ Published online: 16 November 2020 \\ (C) The Author(s) 2020
}

\begin{abstract}
This paper discusses model order reduction of linear time-invariant (LTI) systems over limited frequency intervals within the framework of balanced truncation. Two new frequency-dependent balanced truncation methods are developed, one is singlefrequency $(S F)$-type frequency-dependent balanced truncation to cope with the cases that only a single dominating point of the operating frequency interval is pre-known, and the other is interval-type frequency-dependent balanced truncation to deal with the case that both the upper and lower bounds of the relevant frequency interval are known a priori. Error bounds for both approaches are derived to estimate the approximation error over a pre-specified frequency interval. In contrast to other error bounds for frequency-weighted or frequency-limited balanced truncation, these bounds are given specifically for the interval under consideration and are thus often sharper than the global bounds for previous methods. We show that the new methods generally lead to good in-band approximation performance, and at the same time provide accurate error bounds under certain conditions. Examples are included for illustration.
\end{abstract}

Keywords Model order reduction · Balanced truncation · Linear time-invariant systems $\cdot$ Kalman-Yakubovich-Popov lemma

Mathematics Subject Classification (2010) 93B40 · 93A15 · 93B11

Communicated by: Anthony Nouy

This article belongs to the Topical Collection: Model reduction of parametrized Systems Guest Editors: Anthony Nouy, Peter Benner, Mario Ohlberger, Gianluigi Rozza, Karsten Urban and Karen Willcox

Peter Benner

benner@mpi-magdeburg.mpg.de

Extended author information available on the last page of the article. 


\section{Introduction and problem formulations}

We study model order reduction for linear time-invariant continuous-time systems, where, by abuse of notation, we will denote the system and its transfer function by $G$, and we will use the following representations in state-space, block matrix, and frequency domain form:

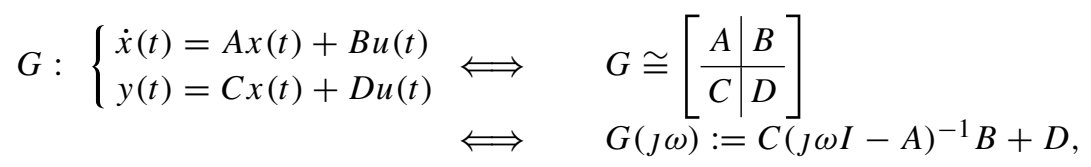

where $A \in \mathbb{C}^{n \times n}, B \in \mathbb{C}^{n \times m}, C \in \mathbb{C}^{p \times n}, D \in \mathbb{C}^{p \times m}$, and at time $t \in[0, \infty]$, $x(t) \in \mathbb{C}^{n}$ is the state vector, $u(t) \in \mathbb{C}^{m}$ is the input signal, and $y(t) \in \mathbb{C}^{p}$ is the output signal. Furthermore, $J:=\sqrt{-1}$ is the imaginary unit and $\omega \in \mathbb{R}$ is the angular frequency in radians per second.

Modeling of complex physical processes frequently leads to large order $n$. The corresponding high storage requirements and expensive computations make it often difficult to simulate or optimize such systems, and may be prohibitive to design a controller for such plant models. In these situations, model order reduction (MOR) becomes an expedient tool; see [1-8] for overviews on MOR techniques and applications. It consists in approximating the system (1) by a reduced-order model (ROM) (or "reduced-order system"):

$$
\begin{array}{r}
G_{r}:\left\{\begin{array}{r}
\dot{x}_{r}(t)=A_{r} x_{r}(t)+B_{r} u(t) \\
y_{r}(t)=C_{r} x_{r}(t)+D_{r} u(t)
\end{array}\right. \\
\Longleftrightarrow G_{r}(\jmath \omega) \cong\left[\begin{array}{c|c}
A_{r} \mid B_{r} \\
\hline C_{r} \mid D_{r}
\end{array}\right] \\
G_{r}(\jmath \omega):=C_{r}\left(\jmath \omega I-A_{r}\right)^{-1} B_{r}+D_{r},
\end{array}
$$

where $A_{r} \in \mathbb{C}^{r \times r}, B_{r} \in \mathbb{C}^{n \times m}, C_{r} \in \mathbb{C}^{p \times n}, D_{r} \in \mathbb{C}^{p \times m}$ with $r \ll n$, and such that $y \approx y_{r}$ for all feasible input signals $u$.

Balanced truncation is a well-studied and the most commonly used MOR scheme in systems and control theory; see [1-3, 5, 9] for details and recent surveys. The standard form is sometimes called Lyapunov balanced truncation, which was first introduced in the systems and control literature by Moore [10]. The prominent advantages of balanced truncation are that it preserves stability and provides an a priori error bound over the entire frequency range as a by-product of the procedure to compute a ROM. In detail, it provides an upper bound of the following entire-frequency (EF) type approximation performance index function:

$$
\sigma_{\max }\left(G(\jmath \omega)-G_{r}(J \omega)\right) \quad \text { for all } \omega \in[-\infty,+\infty],
$$

where $\sigma_{\max }($.$) denotes the maximum singular value of a matrix.$

In many practical applications, the operating frequency of the input signal belongs to a fully or partially known finite-frequency range such as a limited interval (i.e., $\left.\omega \in\left[\varpi_{1}, \varpi_{2}\right]\right)$. For those cases, the ROM is only needed to capture the inputoutput behavior of the original system for input signals with admissible frequency. Correspondingly, good in-band approximation performance is expected, while the out-of-band approximation performance might be neglected; see, e.g., [11-18] for 
such methods. In other words, the objective of finite-frequency (FF) MOR is to minimize the following FF-type performance index function:

$$
\sigma_{\max }\left(G(\jmath \omega)-G_{r}(J \omega)\right) \quad \text { for all } \omega \in\left[\varpi_{1}, \varpi_{2}\right] .
$$

Since standard balanced truncation is intrinsically frequency-independent, we will call it frequency-independent balanced truncation (FIBT) in the sequel. For FIBT, pre-known frequency information cannot be used to further improve the in-band approximation performance (4). To enhance the approximation performance over a pre-specified frequency range, several balancing-related approaches have been developed. Some popular ones include:

1. Singular perturbation approximation (SPA) SPA is a balancing-related method based on the standard FIBT, first introduced by Liu and Anderson [11]. Although FIBT and SPA yield the same EF-type error bound, the characteristics of them complement each other. The ROMs generated by FIBT generally have a smaller error at high frequencies (as the error tends to zero when $\omega$ tends to infinity), and tend to be larger at low frequencies. In contrast, SPA generally leads to good approximation performance at small frequencies as for SPA, $G(0)=G_{r}(0)$ holds. Therefore, SPA is particularly suited for solving MOR problems where good steady-state performance is desired. For a flexible trade-off between the local approximation performance over low-frequency ranges and the global approximation performance over the entire frequency range, a generalized SPA algorithm has been developed by parametrizing the ROM using a user-defined adjustable scalar defining where interpolation is achieved (instead of $\omega=0$ as in standard SPA) [9, Section 1.3].

2. Frequency-weighted balanced truncation (FWBT) In the fields of system analysis and control theory, frequency-weighting functions are conventional tools widely applied for solving various analysis and synthesis problems with pre-known frequency information. For FF-MOR problems, utilizing the frequency-weighting technique and combining it with the standard balanced truncation method is very common. During the last three decades, many frequency-weighted balanced truncation approaches have been developed; see, e.g., [13, 16, 18-21]. The common procedure of FWBT is to build a frequency-weighted model first by introducing input/output frequency-weighted transfer functions and then to apply the standard FIBT to the frequency-weighted model. Indeed, good frequency-specific approximation performance may be obtained if the selected weighting function is an appropriate one. However, the design iterations to search for an appropriate weighting transfer function can be tedious. Besides, FWBT also suffers from the drawback of the increased order of the weighted plant model.

3. Frequency-limited balanced truncation (FLBT) This was first introduced by Gawronski and Juang in [14]. The FLBT methodology stems from changing the definition of the standard Gramians via their integral formulation over the whole imaginary axis to the frequency-limited case, where the integral is limited to the desired frequency interval, and then applying the standard balanced truncation procedure using the frequency-limited Gramians. See, e.g., [15, 17] for enhancements of this technique and [12] for an algorithm applicable to truly large-scale problems with sparse A. A disadvantage of FLBT is that, so far, no 
Table 1 Characterizations of various balancing-related methods

\begin{tabular}{llll}
\hline Assumption & Method & $\begin{array}{l}\text { Error bound desired } \\
\text { for } \sigma_{\max }(E(J \omega))\end{array}$ & $\begin{array}{l}\text { Error bound available } \\
\text { for } \sigma_{\max }(E(J \omega))\end{array}$ \\
\hline EF-MOR & FIBT & $\forall \omega \in[-\infty,+\infty]$ & $\forall \omega \in[-\infty,+\infty]$ \\
$\omega \in[-\infty,+\infty]$ & & $\forall \omega \in\left[0, \varpi_{2}\right]$ & $\forall \omega \in[-\infty,+\infty]$ \\
FF-MOR & SPA & $\forall \omega \in\left[\varpi_{1}, \varpi_{2}\right]$ & $\forall \omega \in[-\infty,+\infty]$ \\
$\omega \in\left[\varpi_{1},+\varpi_{2}\right]$ & FWBT & $\forall \omega \in\left[\varpi_{1}, \varpi_{2}\right]$ & $\forall \omega \in[-\infty,+\infty]$ \\
& FLBT & $\forall \omega \in\left[\varpi_{1}, \varpi_{2}\right]$ & $\forall \omega \in\left[\varpi_{1}, \varpi_{2}\right]$
\end{tabular}

Here, $E(s):=G(s)-G_{r}(s)$ denotes the error system for the ROMs computed by the various approaches

computable error bound is known. To overcome this drawback, several modified FLBT schemes providing error bounds have been proposed [15, 17].

A common feature of the existing finite-frequency balancing-related approaches is that they continue to use the entire-frequency type index (3) to evaluate the actual finite-frequency approximation performance (see Table 1 for an overview).

As illustrated in Table 1, there exists an incompatibility between the intrinsic required and the available error bounds with respect to the existing FF-oriented balancing-related approaches. Since only EF-type error bounds are available, these bounds also bound the error outside the interesting frequency range, and this error can be large as it is of no importance for the method. Thus, these error bounds tend to be very pessimistic w.r.t. predicting the in-band performance of the FF-type methods, and they are often even worse than the standard balanced truncation error bound for FIBT-as FIBT tries to approximate the system over the entire frequency range, no part of the frequency band is neglected by the approximation! Requiring a good approximation over a (small) finite frequency range, one would expect a better error bound than that available for the whole frequency range, and a better true approximation quality in the frequency band of interest. This motivates us to revisit the finite-frequency model reduction problems.

In this paper, we are concerned with the FF MOR problem within the framework of balanced truncation. The main conceptual innovation of this paper can be seen in establishing new methods coming with FF-type error bounds instead of EFtype error bounds to estimate the in-band approximation error. First, we focus on the case that only a single dominating operating frequency point $\varpi$ is pre-known. By exploiting a special class of parameterized Möbius transformations, an SF-type frequency-dependent balanced truncation (FDBT) method is developed based on the generalized Kalman-Yakubovich-Popov (KYP) lemma (developed by Iwasaki and Hara in [22]). It is shown that the proposed SF-type FDBT method provides a scalable SF-type error bound with respect to a user-defined parameter. By adjusting the parameter, satisfactory approximation performance can be obtained. Second, we discuss the cases that both an upper and a lower bound for the operating frequency 
interval are known. Following the same generalized KYP lemma-based methodology as before, an interval-type frequency-dependent balanced truncation method is developed. Moreover, an interval-type error bound for this approach is derived. The interval-type FDBT method generally gives rise to good in-band approximation performance. In particular, we show that a small in-band approximation error with small interval-type error bound can be simultaneously generated as long as the pre-specified interval is small enough.

The remainder of this paper is organized as follows: First, we introduce the generalized KYP lemma in Section 2. Then, we derive the SF-type FDBT method and the interval-type FDBT method in Sections 3 and 4, respectively. Next, we demonstrate the effectiveness and advantages of the proposed methods by several examples in Section 5. Finally, we end with conclusions and an outlook on future work in Section 6.

Notation For a matrix $M, M^{T}$ and $M^{*}$ denote its transpose and conjugate transpose, respectively. The symbol $*$ within a matrix represents entries known due to symmetry. $\mathrm{He}(M)$ denotes $\frac{1}{2}\left(M+M^{*}\right)$, i.e., the Hermitian part of a matrix. $\sigma_{\max }(M)$ represents the maximum singular value of a matrix $M . \operatorname{Re}(x)$ and $\operatorname{Im}(x)$ are the real and imaginary parts of the complex quantity $x$, respectively, and $J:=\sqrt{-1} \cdot M^{\frac{1}{2}}$ is a square root of the matrix $M$ and $M^{\frac{1}{2} \star}$ denotes the positive principle square root of $M$ (i.e., all the eigenvalues of $M^{\frac{1}{2} \star}$ have positive real part). $I$ represents the identity matrix of appropriate dimension. $M>0$ stands for " $M$ is positive definite," while $M \geq 0$ denotes positive semi-definiteness. $I_{n}$, or $I$ if the dimension is clear from the context, is the identity matrix of size $n \times n$.

\section{The fundamental tool}

The KYP lemma [23] is a cornerstone in systems and control theory. In fact, the EF-type error bound provided by the standard FIBT can be proved and interpreted with the aid of the KYP lemma. In [22], Iwasaki and Hara successfully generalized the KYP lemma from the EF case to FF cases. The generalized KYP lemma plays a fundamental role in our development, and therefore it is stated here.

Proposition 1 [Generalized KYP lemma, [22]] Consider the continuous-time system (1), then the following statements are equivalent:

(i) The frequency domain inequality:

$$
\sigma_{\max }(G(J \omega)) \leq \gamma \quad \text { holds for all } \omega \in\left[\omega_{1}, \omega_{2}\right] .
$$

(ii) There exist Hermitian matrices $P$ and $Q>0$ of appropriate dimensions, satisfying the linear matrix inequality:

$$
\left[\begin{array}{cl}
-H e\left(\mathcal{A}\left(\omega_{1}\right) Q \mathcal{A}\left(\omega_{2}\right)^{*}\right)+\mathcal{L}(P) & \mathcal{A}\left(\omega_{c}\right) Q C^{*}+P C^{*}+B D^{*} \\
* & -C Q C^{*}+D D^{*}-\gamma^{2} I
\end{array}\right] \leq 0,
$$

where $\mathcal{L}(P):=A P+P A^{*}+B B^{*}$ and $\mathcal{A}(\omega):=\jmath \omega I-A$ for $\omega \in \mathbb{R}$. 


\section{Frequency-dependent balanced truncation over uncertain frequency interval}

In this section, we focus on the MOR problem for an uncertain frequency interval (i.e., $\omega \in[\varpi-\delta, \varpi+\delta]$, where $\varpi$ denotes the pre-known dominating frequency point, and $\delta$ denotes the unknown size parameter of the frequency interval). First, we construct a class of parameterized frequency-dependent extended systems, which plays an important role in the development of SF-type FDBT. Then, the related results and algorithm are presented.

Definition 1 (SF-type frequency-dependent extended systems) Given an LTI system (1) and a pre-specified frequency point $\varpi$, the $S F$-type frequency-dependent extended systems are constructed as:

$$
\begin{aligned}
G_{\epsilon \varpi}(J \omega) & \cong\left[\begin{array}{c|c}
A_{\epsilon}(\varpi) & B_{\epsilon}(\varpi) \\
\hline C_{\epsilon}(\varpi) & D_{\epsilon}(\varpi)
\end{array}\right] \\
& =\left[\begin{array}{c|c}
J \varpi I-\epsilon(\epsilon I+\mathcal{A}(\varpi))^{-1} \mathcal{A}(\varpi) & \epsilon(\epsilon I+\mathcal{A}(\varpi))^{-1} B \\
\hline \epsilon C(\epsilon I+\mathcal{A}(\varpi))^{-1} & D+C(\epsilon I+\mathcal{A}(\varpi))^{-1} B
\end{array}\right],
\end{aligned}
$$

where $\epsilon>0$ is a user-specified real scalar and $\mathcal{A}($.$) is defined as in Proposition 1$.

In the above definition, $\epsilon$ should be a scalar satisfying the condition:

$$
\epsilon \neq-\left(J \varpi-\lambda_{i}\right)
$$

to ensure the invertibility of $(\epsilon I+\jmath \varpi I-A)$, where $\lambda_{i}, i=1, \ldots, n$, denote the eigenvalues of the matrix $A$.

An interpretation of the transformation used in Definition 1 as a Möbius transformation follows from the following result, which can be proved by simple algebraic manipulations.

Proposition 2 For a given LTI system (1), the corresponding SF-type frequencydependent extended system (7) can be obtained by applying a particular Möbius transformation as follows:

$$
G_{\epsilon \varpi}(\jmath \omega)=G\left(\frac{a \cdot \jmath \omega+b}{c \cdot \jmath \omega+d}\right),
$$

where $a=\epsilon-\jmath \varpi, b=-\varpi^{2}, c=-1, d=\epsilon+\jmath \varpi$.

We obtain the following basic properties.

Proposition 3 The following statements hold.

(a) If the original LTI system (1) is Hurwitz stable and $\epsilon>0$, then the corresponding SF-type frequency-dependent extended system is stable. 
(b) If the original LTI system (1) is unstable with the unstable eigenvalues of $A$ denoted by $\lambda_{i}^{+}, i=1, \ldots, n_{u}$, then the corresponding SF-type frequencydependent extended system is stable if $\epsilon$ satisfies

$$
0<\epsilon<\min _{i=1, \ldots, n_{u}}\left\{\epsilon_{i}^{+}\right\}
$$

where $\epsilon_{i}^{+}=\left(\varpi-\operatorname{Im}\left(\lambda_{i}\right)\right)^{2} / \operatorname{Re}\left(\lambda_{i}\right)+\operatorname{Re}\left(\lambda_{i}\right)$.

Proof 1. Let us denote by $\lambda_{i}, i=1,2, \ldots, n$, and $\lambda_{\epsilon, i}(\varpi), i=1,2, \ldots, n$, the eigenvalues of the matrices $A$ and $A_{\epsilon}(\varpi)$, respectively. According to the mapping between $A$ and $A_{\epsilon}(\varpi)$ given in (7), we know that

$$
\lambda_{\epsilon, i}(\varpi)=\jmath \varpi-\epsilon\left(\jmath \varpi-\lambda_{i}\right)\left(\epsilon+\jmath \varpi-\lambda_{i}\right)^{-1}, \quad i=1, \ldots, n .
$$

Noticing that $\operatorname{Re}\left(\lambda_{i}\right)<0$ if the system $G(J \omega)$ is stable, the following inequality

$$
\operatorname{Re}\left(\lambda_{\epsilon, i}(\varpi)\right)=-\frac{-\epsilon \operatorname{Re}\left(\lambda_{i}\right)\left(\epsilon-\operatorname{Re}\left(\lambda_{i}\right)\right)+\epsilon\left(\varpi-\operatorname{Im}\left(\lambda_{i}\right)\right)^{2}}{\left(\epsilon-\operatorname{Re}\left(\lambda_{i}\right)\right)^{2}+\left(\varpi-\operatorname{Im}\left(\lambda_{i}\right)\right)^{2}}<0, \quad i=1, \ldots, n,
$$

holds if $\epsilon>0$, which proves Part (a).

2. Denote by $\lambda_{\epsilon, i}^{+}(\varpi), i=1, \ldots, n_{u}$, the eigenvalues of $A_{\epsilon}(\varpi)$ mapped from $\lambda_{i}^{+}$, i.e.

$$
\lambda_{\epsilon, i}^{+}(\varpi)=\jmath \varpi-\epsilon\left(\jmath \varpi-\lambda_{i}^{+}\right)\left(\epsilon+\jmath \varpi-\lambda_{i}^{+}\right)^{-1}, \quad i=1, \ldots, n_{u},
$$

then it can be concluded that $\operatorname{Re}\left(\lambda_{\epsilon, i}^{+}(\varpi)\right)<0, i=1, \ldots, n_{u}$, for all $\epsilon$ satisfying $0<\epsilon<\min _{i=1, \ldots, n_{u}}\left\{\epsilon_{i}^{+}\right\}$, according to the formula (8). This completes the proof.

Definition 2 (SF-type frequency-dependent Lyapunov equations) Given a linear continuous-time system (1) and one of its corresponding Hurwitz stable SF-type frequency-dependent extended systems (7, then the following two Lyapunov equations:

$$
\begin{aligned}
& A_{\epsilon}(\varpi) W_{c \epsilon}(\varpi)+W_{c \epsilon}(\varpi) A_{\epsilon}^{*}(\varpi)+B_{\epsilon}(\varpi) B_{\epsilon}^{*}(\varpi)=0, \\
& A_{\epsilon}^{*}(\varpi) W_{o \epsilon}(\varpi)+W_{o \epsilon}(\varpi) A_{\epsilon}(\varpi)+C_{\epsilon}^{*}(\varpi) C_{\epsilon}(\varpi)=0
\end{aligned}
$$

are the SF-type frequency-dependent controllability and observability Lyapunov equations of the continuous-time system (1). Furthermore, their respective solutions $W_{c \epsilon}(\varpi)$ and $W_{o \epsilon}(\varpi)$ will be referred to as SF-type frequency-dependent controllability and observability Gramians of the continuous-time system (1).

Definition 3 (SF-type frequency-dependent balanced realization) Given a linear continuous-time system (1) and one of its Hurwitz stable SF-type frequencydependent extended systems (7). If the corresponding SF-type frequency-dependent controllability and observability Gramians are equal and diagonal, i.e., the following Lyapunov equations:

$$
\begin{aligned}
& A_{\epsilon}(\varpi) \Sigma_{\epsilon}(\varpi)+\Sigma_{\epsilon}(\varpi) A_{\epsilon}^{*}(\varpi)+B_{\epsilon}(\varpi) B_{\epsilon}^{*}(\varpi)=0, \\
& A_{\epsilon}^{*}(\varpi) \Sigma_{\epsilon}(\varpi)+\Sigma_{\epsilon}(\varpi) A_{\epsilon}(\varpi)+C_{\epsilon}^{*}(\varpi) C_{\epsilon}(\varpi)=0
\end{aligned}
$$


simultaneously hold, then this particular realization will be referred to as SF-type frequency-dependent balanced realization.

Proposition 4 Suppose the given system (1) is stable and let $W_{c}, W_{o}, \Sigma$ denote its standard controllability, observability, and balanced Gramian matrices, respectively, then the following statements hold:
1. $W_{c} \geq W_{c \epsilon}(\varpi) \geq 0$,
$W_{o} \geq W_{o \epsilon}(\varpi) \geq 0$,
$\Sigma \geq \Sigma_{\epsilon}(\varpi) \geq 0 ;$
2. $\lim _{\varepsilon \rightarrow 0} W_{c \epsilon}(\varpi)=0$,
$\lim _{\varepsilon \rightarrow 0} W_{o \epsilon}(\varpi)=0$,
$\lim _{\varepsilon \rightarrow 0} \Sigma_{\epsilon}(\varpi)=0$;
3. $\lim _{\varepsilon \rightarrow \infty} W_{c \epsilon}(\varpi)=W_{c}$,
$\lim _{\varepsilon \rightarrow \infty} W_{o \epsilon}(\varpi)=W_{o}$,
$\lim _{\varepsilon \rightarrow \infty} \Sigma_{\epsilon}(\varpi)=\Sigma$.

Proof 1. It is well known (see, e.g., [1, 2, 9, 24]) that the standard controllability and observability Gramian matrices $W_{c}, W_{o}$ of system (1) satisfy the following standard frequency-independent Lyapunov equations:

$$
\begin{aligned}
& A W_{c}+W_{c} A^{*}+B B^{*}=0, \\
& A^{*} W_{o}+W_{o} A+C^{*} C=0 .
\end{aligned}
$$

Now post-and-pre multiply the SF-type frequency-dependent Lyapunov equations (9) by $\epsilon^{-1}(\epsilon I+J \varpi I-A)$ and use the notation from Proposition 1 to obtain:

$$
\begin{aligned}
& A W_{c \epsilon}(\varpi)+W_{c \epsilon}(\varpi) A^{*}-2 \epsilon^{-1} \mathcal{A}(\varpi) W_{c \epsilon}(\varpi) \mathcal{A}(\varpi)^{*}+B B^{*}=0, \\
& A^{*} W_{o \epsilon}(\varpi)+W_{o \epsilon}(\varpi) A-2 \epsilon^{-1} \mathcal{A}(\varpi)^{*} W_{o \epsilon}(\varpi) \mathcal{A}(\varpi)+C^{*} C=0 .
\end{aligned}
$$

Furthermore, the following equations can be derived by subtracting equations in (11) from the corresponding ones in (12):

$$
\begin{aligned}
& A\left(W_{c}-W_{c \epsilon}(\varpi)\right)+\left(W_{c}-W_{c \epsilon}(\varpi)\right) A^{*}+2 \epsilon^{-1} \mathcal{A}(\varpi) W_{c \epsilon}(\varpi) \mathcal{A}(\varpi)^{*}=0, \\
& A^{*}\left(W_{o}-v W_{o \epsilon}(\varpi)\right)+\left(W_{o}-W_{o \epsilon}(\varpi)\right) A+2 \epsilon^{-1} \mathcal{A}(\varpi)^{*} W_{o \epsilon}(\varpi) \mathcal{A}(\varpi)=0 .
\end{aligned}
$$

From standard Lyapunov stability theory (e.g., [25, Chapter 13, Proposition $1]$ ), it follows that $W_{c \epsilon}(\varpi), W_{o \epsilon}(\varpi)$ are positive semidefinite due to stability of $A_{\epsilon}(\varpi)$ (implied by Proposition 3 a) and positive semidefiniteness of $B_{\epsilon}(\varpi) B_{\epsilon}^{*}(\varpi)$ and $C_{\epsilon}(\varpi)^{*} C_{\epsilon}(\varpi)$. Thus, using the same argument from stability theory, we can also conclude that $\left(W_{c}-W_{o \epsilon}(\varpi)\right) \geq 0$ and $\left(W_{o}-W_{o \epsilon}(\varpi)\right) \geq$ 0 since

$$
\frac{2}{\epsilon} \mathcal{A}(\varpi) W_{c \epsilon}(\varpi) \mathcal{A}(\varpi)^{*} \geq 0 \quad \text { and } \quad \frac{2}{\epsilon} \mathcal{A}(\varpi)^{*} W_{o \epsilon}(\varpi) \mathcal{A} \geq 0 .
$$

Thus, the proof for the controllability and observability Gramians is complete. The result for the balanced Gramians follows from that for the two separate Gramians of the original and transformed systems, respectively, by observing that they are equal to $\Sigma$ and $\Sigma_{\epsilon}(\varpi)$ in the balanced case.

2. We prove this for $W_{c \epsilon}(\varpi)$, the existence of the other limits can be shown analogously. Re-writing the first equation in (12) as

$$
\frac{\epsilon}{2}\left(A W_{c \epsilon}(\varpi)+W_{c \epsilon}(\varpi) A^{*}+B B^{*}\right)=\mathcal{A}(\varpi) W_{c \epsilon}(\varpi) \mathcal{A}(\varpi)^{*}
$$


and noting that $W_{c \epsilon}(\varpi)$ is bounded from above and below due to (a), the limit for $\epsilon \rightarrow 0$ of the left hand-side exists and is zero. Hence, the limit of the righthand side exists as well and is zero:

$$
0=\lim _{\epsilon \rightarrow 0} \mathcal{A}(\varpi) W_{c \epsilon}(\varpi) \mathcal{A}(\varpi)^{*}=\mathcal{A}(\varpi)\left(\lim _{\epsilon \rightarrow 0} W_{c \epsilon}(\varpi)\right) \mathcal{A}(\varpi)^{*} .
$$

The result follows by noting that $\mathcal{A}(\varpi)=\jmath \varpi I-A$ is invertible for all $\varpi \in \mathbb{R}$ due to the stability of $A$.

3. It can be easily observed from the identity

$$
\epsilon(\epsilon I+\mathcal{A}(\varpi))^{-1}=I-\mathcal{A}(\varpi)(\epsilon I+\mathcal{A}(\varpi))^{-1}
$$

that the $\varpi$-dependent matrices $A_{\epsilon}(\varpi), B_{\epsilon}(\varpi), C_{\epsilon}(\varpi)$ will recover $A, B, C$ as $\epsilon \rightarrow \infty$, i.e.

$$
\begin{aligned}
& \lim _{\varepsilon \rightarrow \infty} A_{\epsilon}(\varpi)=\lim _{\varepsilon \rightarrow \infty}\left(\jmath \varpi I-\epsilon(\epsilon I+\mathcal{A}(\varpi))^{-1} \mathcal{A}(\varpi)\right)=A, \\
& \lim _{\varepsilon \rightarrow \infty} B_{\epsilon}(\varpi)=\lim _{\varepsilon \rightarrow \infty} \epsilon(\epsilon I+\mathcal{A}(\varpi))^{-1} B=B, \\
& \lim _{\varepsilon \rightarrow \infty} C_{\epsilon}(\varpi)=\lim _{\varepsilon \rightarrow \infty} \epsilon C(\epsilon I+\mathcal{A}(\varpi))^{-1}=C .
\end{aligned}
$$

Then, we can conclude from Eq. (12), using the boundedness of the parameterdependent Gramians due to (a), that

$$
\lim _{\epsilon \rightarrow \infty} W_{c \epsilon}(\varpi)=W_{c}, \quad \lim _{\epsilon \rightarrow \infty} W_{o \epsilon}(\varpi)=W_{o}, \quad \lim _{\epsilon \rightarrow \infty} \Sigma_{\epsilon}(\varpi)=\Sigma .
$$

Two remarks are in order.

Remark 1 The inequalities in Proposition 4 a become strict inequalities if we assume that the original and transformed systems are minimal, i.e., controllable and observable.

Remark 2 The behavior of the parameter-dependent Gramians in their limits indicates that one should choose the parameter $\epsilon$ neither too small nor too large, as they would carry little information for too small $\epsilon$, or no new information compared to the original Gramians for too large $\epsilon$. Nevertheless, there is no quantitative answer on how to choose the parameter that can be deduced from this result.

Now, we are ready to state the main result about SF-type balanced truncation. Note that in order to ease notation, we omit the frequency and parameter dependence of the matrices defining the ROM.

Theorem 1 (SF-type frequency-dependent balanced truncation) Given a linear continuous-time system (1) and the fixed operating frequency $\omega=\varpi$, then for any one of its Hurwitz stable SF-type frequency-dependent extended systems (7) given in balanced realization, i.e.,

$$
\Sigma_{\epsilon}(\varpi)=\operatorname{diag}\left(\Sigma_{\epsilon, 1}(\varpi), \Sigma_{\epsilon, 2}(\varpi)\right)
$$


with

$$
\begin{aligned}
& \Sigma_{\epsilon, 1}(\varpi)=\operatorname{diag}\left(\sigma_{\epsilon, 1}(\varpi), \sigma_{\epsilon, 2}(\varpi), \ldots, \sigma_{\epsilon, r}(\varpi)\right), \\
& \Sigma_{\epsilon, 2}(\varpi)=\operatorname{diag}\left(\sigma_{\epsilon, r+1}(\varpi), \sigma_{\epsilon, r+2}(\varpi), \ldots, \sigma_{\epsilon, n}(\varpi)\right),
\end{aligned}
$$

where $\sigma_{\epsilon, 1}(\varpi) \geq \ldots \geq \sigma_{\epsilon, r}(\varpi)>\sigma_{\epsilon, r+1}(\varpi) \geq \ldots \geq \sigma_{\epsilon n}(\varpi)$, the truncated $r^{\text {th }}$-order model

$$
G_{r}(J \omega) \cong\left[\begin{array}{c|c}
A_{r} & B_{r} \\
\hline C_{r} & D_{r}
\end{array}\right]
$$

is given by (defining $\left.Z_{r}:=\left[\begin{array}{ll}I^{r \times r} & 0^{r \times(n-r)}\end{array}\right]\right)$ :

$$
\begin{aligned}
& A_{r}=\jmath \varpi I-\epsilon Z_{r}\left(\jmath \varpi I-A_{\epsilon}(\varpi)\right) Z_{r}^{T}\left(\epsilon I-Z_{r}\left(\jmath \varpi I-A_{\epsilon}(\varpi)\right) Z_{r}^{T}\right)^{-1}, \\
& B_{r}=\epsilon^{-1}\left(\epsilon I+\jmath \varpi I-A_{r}\right) Z_{r} B_{\epsilon}(\varpi), \\
& C_{r}=\epsilon^{-1} C_{\epsilon}(\varpi) Z_{r}^{T}\left(\epsilon I+\jmath \varpi I-A_{r}\right), \\
& D_{r}=D_{\epsilon}(\varpi)-C_{r}\left(\epsilon I+\jmath \varpi I-A_{r}\right)^{-1} B_{r} .
\end{aligned}
$$

Furthermore, we obtain the following approximation error bounds for $G_{r}$ :

1. SF-type error bound at the given operating frequency $\omega=\varpi$ :

$$
\sigma_{\max }\left(G(\jmath \varpi)-G_{r}(\jmath \varpi)\right) \leq 2 \sum_{i=r+1}^{n} \sigma_{\epsilon, i}(\varpi) .
$$

2. EF-type error bound:

$$
\left\|G-G_{r}\right\|_{\infty} \leq 2 \sum_{i=r+1}^{n} \sigma_{\epsilon, i}(\varpi)+\left\|G-G_{\epsilon \varpi}\right\|_{\infty}+\left\|G_{r}-G_{r, \epsilon \varpi}\right\|_{\infty},
$$

where $\|G\|_{\infty}:=\sup _{\omega \in \mathbb{R}} \sigma_{\max }(G(J \omega))$ is the (Hardy) $H_{\infty}$-norm of a stable rational function $G$ and

$$
\begin{aligned}
G_{r, \epsilon \varpi} & \cong\left[\begin{array}{c|c}
A_{r \epsilon}(\varpi) & B_{r \epsilon}(\varpi) \\
\hline C_{r \epsilon}(\varpi) & D_{r \epsilon}(\varpi)
\end{array}\right] \\
& =\left[\begin{array}{c|c}
J \varpi I-\epsilon\left(\epsilon I+\mathcal{A}_{r}(\varpi)\right)^{-1} \mathcal{A}_{r}(\varpi) & \epsilon\left(\epsilon I+\mathcal{A}_{r}(\varpi)\right)^{-1} B_{r} \\
\hline \epsilon C_{r}\left(\epsilon I+\mathcal{A}_{r}(\varpi)\right)^{-1} & D_{r}+C_{r}\left(\epsilon I+\mathcal{A}_{r}(\varpi)\right)^{-1} B_{r}
\end{array}\right]
\end{aligned}
$$

with $\mathcal{A}_{r}(\omega):=\jmath \omega I-A_{r}$ for $\omega \in \mathbb{R}$.

Proof 1. The detailed proof for $r=n-1$ will be provided in the sequel, and the $r=n-2, \ldots, 1$ cases can then be easily completed step by step.

The error system is realized by

$$
\begin{aligned}
\mathcal{E}_{n}(\jmath \omega) & =G(\jmath \omega)-G_{n-1}(\jmath \omega) \\
& =:\left[\begin{array}{l|l}
\mathcal{A}_{e n} & \mathcal{B}_{e n} \\
\hline \mathcal{C}_{e n} & \mathcal{D}_{e n}
\end{array}\right]=\left[\begin{array}{cc|c}
A_{n-1} & 0 & B_{n-1} \\
0 & A & B \\
\hline-C_{n-1} & C & D-D_{n-1}
\end{array}\right] .
\end{aligned}
$$


From the error system $\mathcal{E}_{n}(J \omega)$, we construct a dilated system $\mathscr{E}_{n}(J \omega)$ as follows:

$$
\mathscr{E}_{n}(\jmath \omega)=\left[\begin{array}{c|c}
\mathscr{A}_{e n} & \mathscr{B}_{e n} \\
\hline \mathscr{C}_{\text {en }} & \mathscr{D}_{\text {en }}
\end{array}\right]=\left[\begin{array}{c|cc}
\mathcal{A}_{\text {en }} & \mathcal{B}_{\text {en }} & \mathcal{B}_{d n} \\
\hline \mathcal{C}_{\text {en }} & \mathcal{D}_{\text {en }} & \mathcal{D}_{d n}^{11} \\
\mathcal{C}_{d n} & \mathcal{D}_{d n}^{12} & \mathcal{D}_{d n}^{22}
\end{array}\right]
$$

where $\mathcal{B}_{d n}, \mathcal{C}_{d n}, \mathcal{D}_{d n}^{12}, \mathcal{D}_{d n}^{21}$, and $\mathcal{D}_{d n}^{22}$ are auxiliary "dilated" matrices, which are constructed as follows:

$$
\begin{aligned}
& \mathcal{B}_{d n}=-\frac{\sigma_{\epsilon n}(\varpi)}{\epsilon}\left(\epsilon I+\jmath \varpi I-\mathcal{A}_{e n}\right)\left[\begin{array}{c}
Z_{n-1} \\
-I
\end{array}\right] \Sigma_{\epsilon}^{-1}(\varpi) C_{\epsilon}(\varpi)^{*}, \\
& \mathcal{C}_{d n}^{*}=-\frac{\sigma_{\epsilon n}(\varpi)}{\epsilon}\left(\epsilon I+\jmath \varpi I-\mathcal{A}_{e n}\right)^{T}\left[\begin{array}{c}
-Z_{n-1} \\
-I
\end{array}\right] \Sigma_{\epsilon}^{-1}(\varpi) B_{\epsilon}(\varpi), \\
& \mathcal{D}_{d n}^{12}=-\mathcal{C}_{e n}\left(\epsilon I+\jmath \varpi I-\mathcal{A}_{e n}\right)^{-1} \mathcal{B}_{d n}+2 \sigma_{\epsilon n}(\varpi) I, \\
& \mathcal{D}_{d n}^{21}=-\mathcal{C}_{d n}\left(\epsilon I+\jmath \varpi I-\mathcal{A}_{e n}\right)^{-1} \mathcal{B}_{e n}+2 \sigma_{\epsilon n}(\varpi) I, \\
& \mathcal{D}_{d n}^{22}=-\mathcal{C}_{d n}\left(\epsilon I+\jmath \varpi I-\mathcal{A}_{e n}\right)^{-1} \mathcal{B}_{d n} .
\end{aligned}
$$

Next, we define the Lyapunov variables $\mathscr{Q}_{e n}=\mathscr{Q}_{e n}^{*} \geq 0$ and $\mathscr{P}_{e n}=\mathscr{P}_{\text {en }}$ as follows:

$$
\begin{aligned}
& \mathscr{Q}_{e n}=\frac{2}{\epsilon}\left[\begin{array}{c}
Z_{n-1} \\
I
\end{array}\right] \Sigma_{\epsilon}(\varpi)\left[\begin{array}{c}
Z_{n-1} \\
I
\end{array}\right]^{T}+\frac{2 \sigma_{\epsilon n}(\varpi)^{2}}{\epsilon}\left[\begin{array}{c}
-Z_{n-1} \\
I
\end{array}\right] \Sigma_{\epsilon}^{-1}(\varpi)\left[\begin{array}{c}
-Z_{n-1} \\
I
\end{array}\right]^{T}, \\
& \mathscr{P}_{e n}=\left[\begin{array}{c}
Z_{n-1} \\
I
\end{array}\right] \Sigma_{\epsilon}(\varpi)\left[\begin{array}{c}
Z_{n-1} \\
I
\end{array}\right]^{T}+\sigma_{\epsilon n}(\varpi)^{2}\left[\begin{array}{c}
-Z_{n-1} \\
I
\end{array}\right] \Sigma_{\epsilon}(\varpi)\left[\begin{array}{c}
-Z_{n-1} \\
I
\end{array}\right]^{T} .
\end{aligned}
$$

Now, we substitute the Lyapunov variables $\mathscr{Q}_{e n}, \mathscr{P}_{e n}$ into the left-hand side of the SF-type matrix inequality (6) for the error system:

$$
\begin{aligned}
& {\left[\begin{array}{cc}
-\left(j \varpi I-\mathscr{A}_{e n}\right) \mathscr{Q}_{e n}\left(J \varpi-\mathscr{A}_{e n}\right)^{*}+\mathscr{L}_{e n}\left(\mathscr{P}_{e n}\right) & \left(\mathrm{j} \varpi I-\mathscr{A}_{e n}\right) \mathscr{C}_{e n}^{*}+\mathscr{P}_{e n} \mathscr{C}_{e n}^{*}+\mathscr{B}_{e n} \mathscr{D}_{e n}^{*} \\
* & -\mathscr{C}_{e n} \mathscr{Q}_{e n} \mathscr{C}_{e n}^{*}+\mathscr{D}_{e n} \mathscr{D}_{e n}^{*}-\left(2 \sigma_{\epsilon n}(\varpi)\right)^{2} I
\end{array}\right]} \\
& =:\left[\begin{array}{cc}
\Pi_{11} & \Pi_{12} \\
* & \Pi_{22}
\end{array}\right]=:\left[\begin{array}{ccc}
\Pi_{11} & \Pi_{12}^{1} & \Pi_{12}^{2} \\
* & \Pi_{22}^{11} & \Pi_{22}^{12} \\
* & * & \Pi_{22}^{22}
\end{array}\right],
\end{aligned}
$$

where $\mathscr{L}_{e n}\left(\mathscr{P}_{e n}\right):=\mathscr{A}_{e n} \mathscr{P}_{e n}+\mathscr{P}_{e n} \mathscr{A}_{e n}^{*}+\mathscr{B}_{e n} \mathscr{B}_{e n}^{*}$. Combined with the balanced SF-type frequency-dependent Lyapunov 10, one can derive the following 
equations for the blocks in the above block matrices:

$$
\begin{aligned}
& \Pi_{11}=-\left(j \varpi I-\mathscr{A}_{e n}\right) \mathscr{Q}_{e n}\left(j \varpi-\mathscr{A}_{e n}\right)^{*}+\mathscr{A}_{e n} \mathscr{P}_{e n}+\mathscr{P}_{e n} \mathscr{A}_{e n}^{*}+\mathscr{B}_{e n} \mathscr{B}_{e n}^{*} \\
& =\left[\frac{1}{\epsilon}\left(\epsilon I+\jmath \varpi I-\mathcal{A}_{e n}\right)\right] \Delta_{1}\left[\frac{1}{\epsilon}\left(\epsilon I+\jmath \varpi I-\mathcal{A}_{e n}\right)\right]^{*}, \\
& \Pi_{12}^{1}=\left(\jmath \varpi I-\mathcal{A}_{e n}\right) \mathscr{Q}_{e n} \mathcal{C}_{e n}^{*}+\mathscr{P}_{e n} \mathcal{C}_{e n}^{*}+\mathcal{B}_{e n} \mathcal{D}_{e n}^{*} \\
& =\left[\epsilon\left(\epsilon I+\jmath \varpi I-\mathcal{A}_{e n}\right)\right] \Delta_{2}\left[\epsilon(\epsilon I+\jmath \varpi I-A)^{-1}\right]^{*} C^{*}, \\
& \Pi_{12}^{2}=\left(\mathrm{J} \varpi I-\mathcal{A}_{e n}\right) \mathscr{Q}_{e n} \mathcal{C}_{e n}^{*}+\mathscr{P}_{e n} \mathcal{C}_{e n}^{*}+\mathcal{B}_{e n} \mathcal{D}_{e n}^{*} \\
& =\left[\epsilon^{-1}\left(\epsilon I+\jmath \varpi I-\mathcal{A}_{e n}\right)\right] \Delta_{3} \Sigma_{\epsilon}^{-1}(\varpi)\left[\epsilon(\epsilon I+\jmath \varpi I-A)^{-1}\right] B, \\
& \Pi_{22}^{11}=-\mathcal{C}_{e n} \mathscr{Q}_{e n} \mathcal{C}_{e n}^{*}+\left[\begin{array}{ll}
\mathcal{D}_{e n} & \mathcal{D}_{d n}^{12}
\end{array}\right]\left[\begin{array}{ll}
\mathcal{D}_{e n} & \mathcal{D}_{d n}^{12}
\end{array}\right]^{*}-\left(2 \sigma_{\epsilon n}(\varpi)\right)^{2} I \\
& =-\mathcal{C}_{e n} \Delta_{2}(\epsilon I+\jmath \varpi I-A)^{-*} C^{*}-C(\epsilon I+\jmath \varpi I-A)^{-1} \Delta_{2}^{*} \mathcal{C}_{e n}{ }^{*}, \\
& \Pi_{22}^{12}=-\mathcal{C}_{e n} \mathscr{Q}_{e n} \mathcal{C}_{e n}^{*}+\left[\begin{array}{ll}
\mathcal{D}_{e n} & \mathcal{D}_{d n}^{12}
\end{array}\right]\left[\begin{array}{ll}
\mathcal{D}_{d n}^{21} & \mathcal{D}_{d n}^{22}
\end{array}\right]^{*} \\
& \left.=-C(\epsilon I+\jmath \varpi I-A)^{-1}\right] \Delta_{2} \mathcal{C}_{d n}^{*}-\sigma_{\epsilon n}(\varpi) \mathcal{C}_{e n} \Delta_{3} \Sigma_{\epsilon}^{-1}(\varpi) \\
& (\epsilon I+\jmath \varpi I-A)^{-1} B, \\
& \Pi_{22}^{22}=-\mathcal{C}_{e n} \mathscr{Q}_{e n} \mathcal{C}_{e n}^{*}+\left[\begin{array}{ll}
\mathcal{D} d n^{21} & \mathcal{D}_{d n}^{22}
\end{array}\right]\left[\begin{array}{ll}
\mathcal{D}_{d n}^{21} & \mathcal{D}_{d n}^{22}
\end{array}\right]^{*}-\left(2 \sigma_{\epsilon n}(\varpi)\right)^{2} I \\
& =-\frac{\sigma_{\epsilon n}^{2}(\varpi)}{\epsilon} B^{*}\left[\epsilon(\epsilon I+\jmath \varpi I-A)^{-1}\right]^{*} \Sigma_{\epsilon}^{-1}(\varpi) \\
& \left(\Delta_{3}+\Delta_{3}^{*}\right) \Sigma_{\epsilon}^{-1}(\varpi)\left[\epsilon(\epsilon I+\jmath \varpi I-A)^{-1}\right] B,
\end{aligned}
$$

where

$$
\begin{aligned}
\Delta_{1}= & {\left[\begin{array}{cc}
A_{r \epsilon}(\varpi) & 0 \\
0 & A_{\epsilon}(\varpi)
\end{array}\right]\left(\left[\begin{array}{cc}
Z_{n-1} \Sigma_{\epsilon}(\varpi) Z_{n-1}^{T} & Z_{n-1} \Sigma_{\epsilon}(\varpi) \\
\Sigma_{\epsilon}(\varpi) Z_{n-1}^{T} & \Sigma_{\epsilon}(\varpi)
\end{array}\right]\right.} \\
+ & \left.\sigma_{\epsilon n}^{2}(\varpi)\left[\begin{array}{cc}
Z_{n-1} \Sigma_{\epsilon}(\varpi)^{-1} Z_{n-1}^{T} & -Z_{n-1} \Sigma_{\epsilon}(\varpi)^{-1} \\
-\Sigma_{\epsilon}(\varpi)^{-1} Z_{n-1}^{T} & \Sigma_{\epsilon}(\varpi)^{-1}
\end{array}\right]\right) \\
+ & \left(\left[\begin{array}{cc}
Z_{n-1} \Sigma_{\epsilon}(\varpi) Z_{n-1}^{T} & Z_{n-1} \Sigma_{\epsilon}(\varpi) \\
\Sigma_{\epsilon}(\varpi) Z_{n-1}^{T} & \Sigma_{\epsilon}(\varpi)
\end{array}\right]\right. \\
+ & \left.\sigma_{\epsilon n}^{2}(\varpi)\left[\begin{array}{cc}
Z_{n-1} \Sigma_{\epsilon}(\varpi)^{-1} Z_{n-1}^{T} & -Z_{n-1} \Sigma_{\epsilon}(\varpi)^{-1} \\
-\Sigma_{\epsilon}(\varpi)^{-1} Z_{n-1}^{T} & \Sigma_{\epsilon}(\varpi)^{-1}
\end{array}\right]\right)\left[\begin{array}{cc}
A_{r \epsilon}(\varpi) & 0 \\
0 & A_{\epsilon}(\varpi)
\end{array}\right] \\
+ & {\left[\begin{array}{c}
Z_{n-1} B_{\epsilon}(\varpi) \\
B_{\epsilon}(\varpi)
\end{array}\right]\left[\begin{array}{c}
Z_{n-1} B_{\epsilon}(\varpi) \\
B_{\epsilon}(\varpi)
\end{array}\right]^{*} }
\end{aligned}
$$




$$
\begin{aligned}
& +\sigma_{\epsilon n}^{2}(\varpi)\left[\begin{array}{c}
Z_{n-1} \Sigma_{\epsilon}(\varpi)^{-1} C_{\epsilon}{ }^{*}(\varpi) \\
\Sigma_{\epsilon}(\varpi)^{-1} C^{*}{ }_{\epsilon}(\varpi)
\end{array}\right]\left[\begin{array}{c}
Z_{n-1} \Sigma_{\epsilon}(\varpi)^{-1} C_{\epsilon}{ }^{*}(\varpi) \\
\Sigma_{\epsilon}(\varpi)^{-1} C^{*}{ }_{\epsilon}(\varpi)
\end{array}\right]^{*} \\
& =0 \text {, } \\
& \Delta_{2}=\left[\begin{array}{cc}
Z_{n-1} \Sigma_{\epsilon}(\varpi) Z_{n-1}^{T} & Z_{n-1} \Sigma_{\epsilon}(\varpi) \\
\Sigma_{\epsilon}(\varpi) Z_{n-1}^{T} & \Sigma_{\epsilon}(\varpi)
\end{array}\right]\left[\begin{array}{c}
-Z_{n-1} \\
I
\end{array}\right] \\
& +\quad \sigma_{\epsilon n}^{2}(\varpi)\left[\begin{array}{cc}
Z_{n-1} \Sigma_{\epsilon}(\varpi)^{-1} Z_{n-1}^{T} & -Z_{n-1} \Sigma_{\epsilon}(\varpi)^{-1} \\
-\Sigma_{\epsilon}(\varpi)^{-1} Z_{n-1}^{T} & \Sigma_{\epsilon}(\varpi)^{-1}
\end{array}\right]\left[\begin{array}{c}
-Z_{n-1} \\
I
\end{array}\right] \\
& +2 \sigma_{\epsilon n}(\varpi)\left[\begin{array}{c}
\sigma_{\epsilon n}(\varpi) Z_{n-1} \Sigma_{\epsilon}(\varpi)^{-1} \\
-\sigma_{\epsilon n}(\varpi) \Sigma_{\epsilon}(\varpi)^{-1}
\end{array}\right] \\
& =0 \text {, } \\
& \Delta_{3}=\left[\begin{array}{cc}
Z_{n-1} \Sigma_{\epsilon}(\varpi) Z_{n-1}^{T} & Z_{n-1} \Sigma_{\epsilon}(\varpi) \\
\Sigma_{\epsilon}(\varpi) Z_{n-1}^{T} & \Sigma_{\epsilon}(\varpi)
\end{array}\right]\left[\begin{array}{c}
-Z_{n-1} \\
-I
\end{array}\right] \\
& +\quad 2 \sigma_{\epsilon n}(\varpi)\left[\begin{array}{c}
\sigma_{\epsilon n}^{-1}(\varpi) Z_{n-1} \Sigma_{\epsilon}(\varpi) \\
\sigma_{\epsilon n}^{-1}(\varpi) \Sigma_{\epsilon}(\varpi)
\end{array}\right] \\
& +\quad \sigma_{\epsilon n}^{2}(\varpi)\left[\begin{array}{cc}
Z_{n-1} \Sigma_{\epsilon}(\varpi)^{-1} Z_{n-1}^{T} & -Z_{n-1} \Sigma_{\epsilon}(\varpi)^{-1} \\
-\Sigma_{\epsilon}(\varpi)^{-1} Z_{n-1}^{T} & \Sigma_{\epsilon}(\varpi)^{-1}
\end{array}\right]\left[\begin{array}{c}
-Z_{n-1} \\
-I
\end{array}\right] \\
& =0 \text {. }
\end{aligned}
$$

Now, according to the generalized KYP lemma (Proposition 1), the dilated error system $\mathscr{E}_{n}(\mathrm{~J} \omega)$ satisfies:

$$
\left\|\mathscr{E}_{n}(\mathrm{~J} \varpi)\right\|_{\infty} \leq 2 \sigma_{\epsilon, n}(\varpi) .
$$

Therefore, for the error system $\mathcal{E}_{n}(J \omega)$, it holds that

$$
\left\|\mathcal{E}_{n}(J \varpi)\right\|_{\infty} \leq\left\|\mathscr{E}_{n}(J \varpi)\right\|_{\infty} \leq 2 \sigma_{\epsilon, n}(\varpi)
$$

This completes the proof SF-type error bound (17) for $r=n-1$. The remainder of the proof for the $r=n-2, \ldots, 1$ can be easily completed in a recursive way.

2. From Eqs. 16 and 19, it can be concluded that the SF-type frequency-dependent extended system $G_{r \in \varpi}(J \omega)$ of the reduced system $G_{r}(j \omega)$ can be obtained by applying the standard FIBT algorithm for $G_{\epsilon \varpi}(J \omega)$. Therefore, we have:

$$
\sigma_{\max }\left(G_{\epsilon \varpi}(J \omega)-G_{r \epsilon \varpi}(J \omega)\right) \leq 2 \sum_{i=r+1}^{n} \sigma_{\epsilon, i}(\varpi), \quad \text { for all } \omega \in[-\infty,+\infty] .
$$

Noting that

$$
G(J \omega)-G_{r}(J \omega)=\left(G_{\epsilon \varpi}(J \omega)-G_{r \epsilon \varpi}(J \omega)\right)+\left(G(J \omega)-G_{\epsilon \varpi}(J \omega)\right)+\left(G_{r \epsilon \varpi}(J \omega)-G_{r}(\jmath \omega)\right)
$$


and using the triangle inequality, we get:

$$
\begin{aligned}
& \sigma_{\max }\left(G(J \omega)-G_{r}(J \omega)\right) \\
& \leq \sigma_{\max }\left(G_{\epsilon \varpi}(J \omega)-G_{r \epsilon \varpi}(J \omega)\right)+\sigma_{\max }\left(G(J \omega)-G_{\epsilon \varpi}(J \omega)\right) \\
&+\sigma_{\max }\left(G_{r}(J \omega)-G_{r \epsilon \varpi}(J \omega)\right) \\
& \leq 2 \sum_{i=r+1}^{n} \sigma_{\epsilon, i}(\varpi)+\left\|G(J \omega)-G_{\epsilon \varpi}(J \omega)\right\|_{\infty}+\left\|G_{r}(J \omega)-G_{r \epsilon \varpi}(J \omega)\right\|_{\infty}
\end{aligned}
$$

for all $\omega \in[-\infty,+\infty]$. This completes the proof of the entire-frequency error bound (18).

Based on the above results, we are now able to present the SF-type frequencydependent balanced truncation algorithm, shown in Algorithm 1.

\section{Algorithm 1 SF-type FDBT.}

Input: Full-order model $(A, B, C, D)$, frequency $\varpi$, user-defined parameter $\epsilon$ and the desired order of the ROM, $r$.

Output: ROM $\left(A_{r}, B_{r}, C_{r}, D_{r}\right)$.

Step 1. Solve the SF-type frequency-dependent Lyapunov equations (9)

Step 2. Get the SF-type frequency-dependent balanced realization of the given system by coordinate transformation:

$$
\begin{aligned}
& {\left[\begin{array}{c|c}
A_{\epsilon b}(\varpi) & B_{\epsilon b}(\varpi) \\
\hline C_{\epsilon b}(\varpi) & D_{\epsilon b}(\varpi)
\end{array}\right]} \\
& =\left[\begin{array}{c|c}
T_{\epsilon}^{-1}(\varpi) A_{\epsilon}(\varpi) T_{\epsilon}(\varpi) & T_{\epsilon}^{-1}(\varpi) B_{\epsilon}(\varpi) \\
\hline C_{\epsilon}(\varpi) T_{\epsilon}(\varpi) & \left.D_{\epsilon}(\varpi)+C_{\epsilon b}(\varpi)(\epsilon+\jmath \varpi) I-A_{\epsilon b}(\varpi)\right)^{-1} B_{\epsilon b}(\varpi)
\end{array}\right],
\end{aligned}
$$

where $T_{\epsilon}(\varpi)$ is the matrix that simultaneously diagonalizes the matrices $W_{c \epsilon}(\varpi)$ and $W_{o \epsilon}(\varpi)$, i.e.,

$$
T_{\epsilon}^{-1}(\varpi) W_{c \epsilon}(\varpi) T_{\epsilon}(\varpi)=T_{\epsilon}^{*}(\varpi) W_{o \epsilon}(\varpi) T_{\epsilon}^{-*}(\varpi)=\Sigma_{\epsilon}(\varpi) .
$$

Step 3. Compute the reduced-order model as:

$$
\begin{aligned}
A_{r} & =\jmath \varpi I-\epsilon Z_{r}\left(j \varpi I-A_{\epsilon b}(\varpi)\right) Z_{r}^{T}\left(\epsilon I-Z_{r}\left(\jmath \varpi I-A_{\epsilon b}(\varpi)\right) Z_{r}^{T}\right)^{-1}, \\
B_{r} & =\frac{1}{\epsilon}\left((\epsilon+\jmath \varpi) I-A_{r}\right) Z_{r} B_{\epsilon b}(\varpi), \\
C_{r} & =\frac{1}{\epsilon} C_{\epsilon b}(\varpi) Z_{r}^{T}\left((\epsilon+\jmath \varpi) I-A_{r}\right), \\
D_{r} & =D_{\epsilon b}(\varpi)-C_{r}\left((\epsilon+\jmath \varpi) I-A_{r}\right)^{-1} B_{r} .
\end{aligned}
$$


Some remarks are in order.

Remark 3 According to Proposition 4, the SF-type error bound can be regulated to an arbitrary small value by decreasing the parameter $\epsilon$. In other words, arbitrary approximation accuracy at the given frequency point $\omega=\varpi$ can be obtained. In order to achieve satisfactory approximation performance in the neighboring intervals $(\omega \in[\varpi-\delta, \varpi+\delta])$, the value of the parameter $\epsilon$ should be selected carefully. One possible way to pick an appropriate value of $\epsilon$ is to plot the graphs of the SFtype error bound (17) and the EF-type error bound (18) with respect to the parameter $\epsilon$, then one can choose a proper value $\epsilon^{*}$ trading off the SF-type and EF-type error against each other. Furthermore, it is suggested to choose the value of $\epsilon$ to be smaller than $\epsilon^{*}$ if there exists an estimate $(\hat{\delta})$ on the size of the uncertain frequency interval. The smaller $\hat{\delta}$ is, the smaller value of $\epsilon$ can be chosen in general.

Remark 4 For the sake of theoretical completeness, the SF-type FDBT approach is developed in a complex setting. The original system matrices and the reduced system matrices are allowed to be complex. This is not an issue if the ROM is used, e.g., in frequency-response analysis where complex data are generated anyway. In this situation, only a good approximation quality and the fast evaluation of the ROM are of importance. In other applications like transient simulations or feedback control design, only realizations with real system matrices may be of practical interest. Restricting the ROM to be real, the proposed SF-type FDBT can only be applied in the case that $\varpi=0$. It is easy to find that the involved matrices $W_{c \epsilon}(\varpi), W_{o \epsilon}(\varpi), T_{\epsilon}(\varpi)$ and the generated ROM $\left(A_{r}, B_{r}, C_{r}, D_{r}\right)$ are all real if the original system is real and the frequency point is $\varpi=0$. In the framework of balancing-related methods, the proposed SF-type FDBT is not the only way for solving model order reduction problems assuming the dominating frequency is $\varpi=0$. As discussed in Section 1, SPA is also regarded as an effective way for improving the approximation performance over low-frequency ranges. However, it should be noticed that the underlying mechanisms and the algorithms of SPA and SF-type FDBT are totally different. Which one will perform better regarding the low-frequency approximation accuracy depends on the given original system model. From the results of Example 3 in Section 5, to say the least, the proposed SF-type FDBT can be viewed as a viable alternative option besides SPA.

Remark 5 It is well-known that the conventional balanced truncation methods (such as the abovementioned FIBT, SPA, FWBT, and FGBT) are developed for stable systems. To make those methods applicable for unstable system, techniques based essentially on decomposing the system in its stable and unstable parts can be employed; see [26-29] for different approaches to achieve this. According to Proposition 3, one can always find a stable SF-type frequency-dependent extended system by choosing a proper $\epsilon$, even if the given original system is unstable. Thus, the SFtype FDBT can be used for coping with model reduction of unstable systems directly. The price to be paid is that stability of the ROM cannot be guaranteed even if the original system is stable. 


\section{Frequency-dependent balanced truncation over known frequency intervals}

In this section, we present our results for the cases that the operating frequency belongs to a pre-known limited interval, i.e., $\omega \in\left[\varpi_{1}, \varpi_{2}\right]$. We will present some necessary definitions first and then show the related results and the interval-type frequency-dependent balanced truncation algorithm.

Definition 4 (Interval-type frequency-dependent extend systems) Given a linear continuous-time system as in (1) and a pre-known frequency interval $\left[\varpi_{1}, \varpi_{2}\right]$, an interval-type frequency-dependent extended system is constructed as follows:

$$
G_{\varpi_{1}, \varpi_{2}}(J \omega) \cong\left[\begin{array}{c|c}
A\left(\varpi_{1}, \varpi_{2}\right) & B\left(\varpi_{1}, \varpi_{2}\right) \\
\hline C\left(\varpi_{1}, \varpi_{2}\right) & D\left(\varpi_{1}, \varpi_{2}\right)
\end{array}\right],
$$

where

$$
\begin{aligned}
& A\left(\varpi_{1}, \varpi_{2}\right)=A, \\
& B\left(\varpi_{1}, \varpi_{2}\right)=\left[\varpi_{d}^{2}\left(\jmath \varpi_{1} I-A\right)^{-1}\left(\jmath \varpi_{2} I-A\right)^{-1}\right]^{\frac{1}{2} \star} B, \\
& C\left(\varpi_{1}, \varpi_{2}\right)=C\left[\varpi_{d}^{2}\left(\jmath \varpi_{1} I-A\right)^{-1}\left(\jmath \varpi_{2} I-A\right)^{-1}\right]^{\frac{1}{2} \star}, \\
& D\left(\varpi_{1}, \varpi_{2}\right)=D+C\left[\left(\jmath \varpi_{c} I-A\right)\left(\jmath \varpi_{1} I-A\right)^{-1}\left(\jmath \varpi_{2} I-A\right)\right]^{-1} B,
\end{aligned}
$$

and

$$
\varpi_{d}=\left(\varpi_{2}-\varpi_{1}\right) / 2, \quad \varpi_{c}=\left(\varpi_{2}+\varpi_{1}\right) / 2 .
$$

Definition 5 (Interval-type frequency-dependent Lyapunov equations) Given a linear continuous-time system as in (1) and a pre-specified frequency interval $\left[\varpi_{1}, \varpi_{2}\right]$, then the following two Lyapunov equations:

$$
\begin{aligned}
& A\left(\varpi_{1}, \varpi_{2}\right) W_{c}\left(\varpi_{1}, \varpi_{2}\right)+W_{c}\left(\varpi_{1}, \varpi_{2}\right) A^{*}\left(\varpi_{1}, \varpi_{2}\right)+B\left(\varpi_{1}, \varpi_{2}\right) B^{*}\left(\varpi_{1}, \varpi_{2}\right)=0, \\
& A^{*}\left(\varpi_{1}, \varpi_{2}\right) W_{o}\left(\varpi_{1}, \varpi_{2}\right)+W_{o}\left(\varpi_{1}, \varpi_{2}\right) A\left(\varpi_{1}, \varpi_{2}\right)+C^{*}\left(\varpi_{1}, \varpi_{2}\right) C\left(\varpi_{1}, \varpi_{2}\right)=0
\end{aligned}
$$

are called interval-type frequency-dependent controllability and observability Lyapunov equations of the continuous-time system (1). Furthermore, the solutions $W_{c}\left(\varpi_{1}, \varpi_{2}\right)$ and $W_{o}\left(\varpi_{1}, \varpi_{2}\right)$ are referred to as interval-type frequency-dependent controllability and observability Gramians of the continuous-time system (1).

Definition 6 (Interval-type frequency-dependent balanced realization) Given a linear continuous-time system (1) and a pre-specified frequency interval $\left[\varpi_{1}, \varpi_{2}\right]$. If the corresponding interval-type frequency-dependent controllability and observability Gramians in Eq. (22) are equal and diagonal, i.e., the following Lyapunov equations

$$
\begin{aligned}
& \left.A\left(\varpi_{1}, \varpi_{2}\right) \Sigma\left(\varpi_{1}, \varpi_{2}\right)+\Sigma\left(\varpi_{1}, \varpi_{2}\right) A_{(}^{*} \varpi_{1}, \varpi_{2}\right)+B\left(\varpi_{1}, \varpi_{2}\right) B^{*}\left(\varpi_{1}, \varpi_{2}\right)=0, \\
& A^{*}\left(\varpi_{1}, \varpi_{2}\right) \Sigma\left(\varpi_{1}, \varpi_{2}\right)+\Sigma\left(\varpi_{1}, \varpi_{2}\right) A\left(\varpi_{1}, \varpi_{2}\right)+C^{*}\left(\varpi_{1}, \varpi_{2}\right) C\left(\varpi_{1}, \varpi_{2}\right)=0
\end{aligned}
$$

hold simultaneously, then this particular realization will be referred to as interval-type frequency-dependent balanced realization. 
For this setup, we can now state the first main result.

Theorem 2 (Interval-type frequency-dependent balanced truncation) Given a linear continuous-time system as in (1) with a pre-specified frequency interval [ $\left.\varpi_{1}, \varpi_{2}\right]$, and assume the system is given by a interval-type frequency-dependent balanced realization with respect to the interval-type frequency-dependent Gramian:

$\Sigma\left(\varpi_{1}, \varpi_{2}\right)=\operatorname{diag}\left(\sigma_{1}\left(\varpi_{1}, \varpi_{2}\right), \ldots, \sigma_{r}\left(\varpi_{1}, \varpi_{2}\right), \sigma_{r+1}\left(\varpi_{1}, \varpi_{2}\right), \ldots, \sigma_{n}\left(\varpi_{1}, \varpi_{2}\right)\right)$,

with

$$
\sigma_{1}\left(\varpi_{1}, \varpi_{2}\right) \geq \ldots \geq \sigma_{r}\left(\varpi_{1}, \varpi_{2}\right)>\sigma_{r+1}\left(\varpi_{1}, \varpi_{2}\right) \geq \ldots \geq \sigma_{n}\left(\varpi_{1}, \varpi_{2}\right) .
$$

Let the $r^{\text {th }}$-order ROM:

$$
G_{r}(J \omega) \cong\left[\begin{array}{c|c}
A_{r} & B_{r} \\
\hline C_{r} & D_{r}
\end{array}\right]
$$

be defined via

$$
\begin{aligned}
& A_{r}=Z_{r} A Z_{r}^{T}, \\
& B_{r}=\left[\varpi_{d}^{2}\left(\jmath \varpi_{1} I-A_{r}\right)^{-1}\left(\jmath \varpi_{2} I-A_{r}\right)^{-1}\right]^{-\frac{1}{2} \star} Z_{r} B\left(\varpi_{1}, \varpi_{2}\right), \\
& C_{r}=C\left(\varpi_{1}, \varpi_{2}\right) Z_{r}^{T}\left[\varpi_{d}^{2}\left(\jmath \varpi_{1} I-A_{r}\right)^{-1}\left(j \varpi_{2} I-A_{r}\right)^{-1}\right]^{-\frac{1}{2} \star}, \\
& D_{r}=D\left(\varpi_{1}, \varpi_{2}\right)-C_{r}\left[\left(\jmath \varpi_{c} I-A\right)\left(j \varpi_{1} I-A_{r}\right)^{-1}\left(j \varpi_{2} I-A_{r}\right)\right]^{-1} B_{r},
\end{aligned}
$$

where $Z_{r}=\left[\begin{array}{ll}I^{r \times r} & 0^{r \times(n-r)}\end{array}\right]$. Then, the truncated model $G_{r}(J \omega)$ has the following properties:

(a) If the original system is stable then the ROM is stable.

(b) The approximation error between the original system model (1) and the truncated $r^{\text {th }}$-order ROM (24) satisfies the following interval-type error bound in the given frequency interval:

$$
\sigma_{\max }\left(G(j \omega)-G_{r}(j \omega)\right) \leq \sum_{i=r+1}^{n} \sqrt{\eta_{i}\left(\varpi_{1}, \varpi_{2}\right)} \quad \text { for all } \quad \omega \in\left[\varpi_{1}, \varpi_{2}\right]
$$

where

$$
\begin{aligned}
\eta_{i}\left(\varpi_{1}, \varpi_{2}\right)= & \sigma_{\max }\left(\left(2 \sigma_{i}\left(\varpi_{1}, \varpi_{2}\right)\right)^{2} I+\ldots\right. \\
& \left.\ldots H e\left(-\mathscr{C}_{e i} \mathcal{N}_{e i} \mathscr{B}_{e i} H e\left(\left[\begin{array}{l}
0 \\
I
\end{array}\right] 2 \sigma_{i}\left(\varpi_{1}, \varpi_{2}\right)\left[\begin{array}{ll}
I & 0
\end{array}\right]\right)\right)\right)
\end{aligned}
$$


and

$$
\begin{aligned}
& \mathscr{B}_{e i}=\left[\begin{array}{ll}
\mathcal{B}_{e i} & \mathcal{B}_{d i}
\end{array}\right] \\
& =\left[\mathcal{M}_{e i}^{-1}\left[\begin{array}{c}
Z_{i-1} \\
Z_{i}
\end{array}\right] B\left(\varpi_{1}, \varpi_{2}\right) \sigma_{i}\left(\varpi_{1}, \varpi_{1}\right) \mathcal{M}_{e i}^{-1} \Sigma_{e i}^{-1}\left[\begin{array}{c}
Z_{i-1} \\
-Z_{i}
\end{array}\right] C^{*}\left(\varpi_{1}, \varpi_{2}\right)\right], \\
& \mathscr{C}_{e i}^{*}=\left[\mathcal{C}_{e i}^{*} \mathcal{C}_{d i}^{*}\right] \\
& =\left[\mathcal{M}_{e i}^{-*}\left[\begin{array}{c}
Z_{i-1} \\
-Z_{i}
\end{array}\right] C^{*}\left(\varpi_{1}, \varpi_{2}\right) \sigma_{i}\left(\varpi_{1}, \varpi_{2}\right) \mathcal{M}_{e i}^{-*} \Sigma_{e i}^{-1}\left[\begin{array}{c}
Z_{i-1} \\
Z_{i}
\end{array}\right] B\left(\varpi_{1}, \varpi_{2}\right)\right], \\
& \mathcal{N}_{e i}=\operatorname{diag}\left(N_{i-1}, N_{i}\right) \\
& =\operatorname{diag}\left(\left[\left(J \varpi_{c} I-A_{i-1}\right)\left(j \varpi_{1} I-A_{i-1}\right)^{-1}\left(j \varpi_{2} I-A_{i-1}\right)^{-1}\right],\right. \\
& \left.\left[\left(J \varpi_{c} I-A_{i}\right)\left(J \varpi_{1} I-A_{i}\right)^{-1}\left(J \varpi_{2} I-A_{i}\right)^{-1}\right]\right), \\
& \mathcal{M}_{e i}=\operatorname{diag}\left(M_{i-1}, M_{i}\right) \\
& =\operatorname{diag}\left(\left[\varpi_{d}^{2}\left(j \varpi_{1} I-A_{i-1}\right)^{-1}\left(j \varpi_{2} I-A_{i-1}\right)^{-1}\right]^{\frac{1}{2}},\right. \\
& \left.\left[\varpi_{d}^{2}\left(\jmath \varpi_{1} I-A_{i}\right)^{-1}\left(\jmath \varpi_{2} I-A_{i}\right)^{-1}\right]^{\frac{1}{2}}\right), \\
& \left.\left.\Sigma_{e i}=\operatorname{diag}\left(\Sigma_{i-1}\left(\varpi_{1}, \varpi_{2}\right), \Sigma_{i}\left(\varpi_{1}, \varpi_{2}\right)\right)\right\}\right) \\
& =\operatorname{diag}\left(Z_{i-1} \Sigma\left(\varpi_{1}, \varpi_{2}\right) Z_{i-1}^{T}, Z_{i} \Sigma\left(\varpi_{1}, \varpi_{2}\right) Z_{i}^{T}\right) .
\end{aligned}
$$

(c) The approximation error between the original system (1) and the $r^{\text {th }}$-order ROM (24) over the entire frequency range satisfies the following EF-type error bound:

$$
\begin{gathered}
\sigma_{\max }\left(G(J \omega)-G_{r}(J \omega)\right) \leq 2 \sum_{i=r+1}^{n} \sigma_{i}\left(\varpi_{1}, \varpi_{2}\right)+\left\|G(J \omega)-G_{\varpi_{1}, \varpi_{2}}(j \omega)\right\|_{\infty} \\
+\left\|G_{r}(J \omega)-G_{r \varpi_{1}, \varpi_{2}}(J \omega)\right\|_{\infty}
\end{gathered}
$$

for all $\omega \in[-\infty,+\infty]$, where $G_{r \varpi_{1}, \varpi_{2}}(j \omega)$ represents the corresponding interval-type frequency-dependent extended system corresponding to $G_{r}(\mathrm{~J} \omega)$, i.e.,

$$
G_{r, \varpi_{1}, \varpi_{2}}(J \omega) \cong\left[\begin{array}{c|c}
A_{r}\left(\varpi_{1}, \varpi_{2}\right) & B_{r}\left(\varpi_{1}, \varpi_{2}\right) \\
\hline C_{r}\left(\varpi_{1}, \varpi_{2}\right) & D_{r}\left(\varpi_{1}, \varpi_{2}\right)
\end{array}\right],
$$

with

$$
\begin{aligned}
A_{r}\left(\varpi_{1}, \varpi_{2}\right) & =A_{r}=Z_{r} A\left(\varpi_{1}, \varpi_{2}\right) Z_{r}^{T}, \\
B_{r}\left(\varpi_{1}, \varpi_{2}\right) & =\left[\varpi_{d}^{2}\left(\jmath \varpi_{1} I-A_{r}\right)^{-1}\left(\jmath \varpi_{2} I-A_{r}\right)^{-1}\right]^{\frac{1}{2} \star} B_{r}=Z_{r} B\left(\varpi_{1}, \varpi_{2}\right), \\
C_{r}\left(\varpi_{1}, \varpi_{2}\right) & =C_{r}\left[\varpi_{d}^{2}\left(\jmath \varpi_{1} I-A_{r}\right)^{-1}\left(j \varpi_{2} I-A_{r}\right)^{-1}\right]^{\frac{1}{2} \star}=C\left(\varpi_{1}, \varpi_{2}\right) Z_{r}^{T}, \\
D_{r}\left(\varpi_{1}, \varpi_{2}\right) & =D_{r}+C_{r}\left[\left(j \varpi_{c} I-A_{r}\right)\left(j \varpi_{1} I-A_{r}\right)^{-1}\left(J \varpi_{2} I-A_{r}\right)\right]^{-1} B_{r} \\
& =D\left(\varpi_{1}, \varpi_{2}\right) .
\end{aligned}
$$

Proof (a) This part can be easily proved as in the proof of stability preservation for classic FIBT; see, e.g., [24]. 
(b) Similar to the proof of the SF-type error bound provided in Theorem 1, we only sketch the proof for the case $r=n-1$. The error system $\mathcal{E}_{n}(J \omega)$ expressing the difference between the original system $G(j \omega)$ and the $(n-1)$ st-order system $G_{n-1}(j \omega)$ can be represented as:

$$
\begin{aligned}
\mathcal{E}_{n}(\jmath \omega) & =G_{n}(\jmath \omega)-G_{n-1}(\jmath \omega)=G(\jmath \omega)-G_{n-1}(\jmath \omega) \\
& \cong\left[\begin{array}{c|c}
\mathcal{A}_{e n} & \mathcal{B}_{e n} \\
\hline \mathcal{C}_{e n} & \mathcal{D}_{e n}
\end{array}\right]=\left[\begin{array}{cc|c}
A_{n-1} & 0 & B_{n-1} \\
0 & A_{n} & B_{n} \\
\hline-C_{n-1} & C_{n} & D_{n}-D_{n-1}
\end{array}\right] .
\end{aligned}
$$

Based on the error system $\mathcal{E}_{n}(J \omega)$, we construct a dilated system $\mathscr{E}_{n}(J \omega)$ as follows:

$$
\begin{aligned}
& \mathscr{E}_{n}(J \omega) \cong\left[\begin{array}{c|c}
\mathscr{A}_{e n} & \mathscr{B}_{\text {en }} \\
\hline \mathscr{C}_{\text {en }} & \mathscr{D}_{\text {en }}
\end{array}\right] \\
& =\left[\begin{array}{cc|c}
\mathcal{A}_{e n} & \mathcal{B}_{e n} & \mathcal{B}_{d n} \\
\hline \mathcal{C}_{e n} & \mathcal{D}_{e n} & -\mathcal{C}_{e n} \mathcal{N}_{e n} \mathcal{B}_{d n}+2 \sigma_{n}\left(\varpi_{1}, \varpi_{2}\right) I \\
\mathcal{C}_{d n}-\mathcal{C}_{d n} \mathcal{N}_{e n} \mathcal{B}_{e n}+2 \sigma_{n}\left(\varpi_{1}, \varpi_{2}\right) I & -\mathcal{C}_{d n} \mathcal{N}_{e n} \mathcal{B}_{d n}
\end{array}\right],
\end{aligned}
$$

where $\mathcal{B}_{e n}, \mathcal{B}_{d n}, \mathcal{C}_{e n}, \mathcal{C}_{d n}$, and $\mathcal{N}_{e n}$ are defined as in Eq. (26)-Eq. (30). Next, we choose two symmetric Lyapunov variables $\mathscr{Q}_{e n}=\mathscr{Q}_{e n}^{*} \geq 0$ and $\mathscr{P}_{e n}=\mathscr{P}_{e n}^{*}$ as follows:

$$
\mathscr{Q}_{e n}=\mathcal{N}_{e n}\left(\omega_{1}, \omega_{2}\right) \mathcal{B}_{e n} \mathcal{B}_{e n}^{*} \mathcal{N}_{e n}\left(\omega_{1}, \omega_{2}\right)^{*}+\mathcal{N}_{e n}\left(\omega_{1}, \omega_{2}\right) \mathcal{B}_{d n} \mathcal{B}_{d n}^{*} \mathcal{N}_{e n}\left(\omega_{1}, \omega_{2}\right)^{*}
$$

and

$$
\begin{aligned}
& \mathscr{P}_{\text {en }}=H e\left(\left(\jmath \omega_{d}\right)\left(\jmath \omega_{1} I-\mathcal{A}_{e n}\right)^{-1} \mathcal{B}_{e n} \mathcal{B}_{e n}^{*}\left(\jmath \omega_{2} I-\mathcal{A}_{e n}\right)^{-*}\right) \\
& +H e\left(\left(\jmath \omega_{d}\right)\left(J \omega_{1} I-\mathcal{A}_{e n}\right)^{-1} \mathcal{B}_{d n} \mathcal{B}_{d n}^{*}\left(J \omega_{2} I-\mathcal{A}_{e n}\right)^{-*}\right) \\
& -\omega_{d}^{2} H e\left(\left(J \omega_{1} I-\mathcal{A}_{e n}\right)^{-1} \mathcal{M}_{e n}^{-1}\left[\begin{array}{c}
Z_{n-1} \\
I
\end{array}\right] \Sigma\left(\omega_{1}, \omega_{2}\right)\left[Z_{n-1}^{T} I\right]\right. \\
& \left.\mathcal{M}_{\text {en }}^{-*}\left(J \omega_{2} I-\mathcal{A}_{\text {en }}\right)^{-*}\right) \\
& -\sigma_{n}^{2} \omega_{d}^{2} H e\left(\left(j \omega_{1} I-\mathcal{A}_{e n}\right)^{-1} \mathcal{M}_{e n}^{-1}\left[\begin{array}{c}
-Z_{n-1} \\
I
\end{array}\right] \Sigma\left(\omega_{1}, \omega_{2}\right)^{-1}\left[-Z_{n-1}^{T} I\right]\right. \\
& \left.\mathcal{M}_{e n}^{-*}\left(J \omega_{2} I-\mathcal{A}_{e n}\right)^{-*}\right) \text {. }
\end{aligned}
$$

Combining the interval-type balanced frequency-dependent Lyapunov (23) and following the proof of Theorem 1, one can derive the inequality:

$$
\begin{aligned}
& {\left[\begin{array}{cc}
-H e\left(\mathscr{R}_{e n}\left(\varpi_{1}\right) \mathscr{Q}_{e n} \mathscr{R}_{e n}\left(\varpi_{2}\right)^{*}\right)+\mathscr{L}_{e n}\left(\mathscr{P}_{e n}\right) & \mathscr{R}_{e n}\left(\varpi_{c}\right) \mathscr{C}_{e n}^{*}+\mathscr{P}_{e n} \mathscr{C}_{e n}^{*}+\mathscr{B}_{e n} \mathscr{D}_{e n}^{*} \\
* & -\mathscr{C}_{e n} \mathscr{Q}_{e n} \mathscr{C}_{e n}^{*}+\mathscr{D}_{e n} \mathscr{D}_{e n}^{*}-\eta_{n} I
\end{array}\right]} \\
& =\left[\begin{array}{cc}
0 & 0 \\
0 & \left(\left(2 \sigma_{n}\left(\varpi_{1}, \varpi_{2}\right)\right)^{2}-\eta_{n}\left(\varpi_{1}, \varpi_{2}\right)\right) I+\mathscr{T}_{\text {en }}
\end{array}\right] \\
& \leq 0 \text {, }
\end{aligned}
$$


where $\mathscr{L}_{e n}\left(\mathscr{P}_{e n}\right)=\mathscr{A}_{e n} \mathscr{P}_{e n}+\mathscr{P}_{e n} \mathscr{A}_{e n}^{*}+\mathscr{B}_{e n} \mathscr{B}_{e n}^{*}, \mathscr{R}_{e n}(\omega)=j \omega I-\mathscr{A}_{e n}$, and

$$
\mathscr{T}_{e n}=H e\left(-\mathscr{C}_{e i} \mathcal{N}_{e i} \mathscr{B}_{e i} H e\left(\left[\begin{array}{l}
0 \\
I
\end{array}\right]\left(2 \sigma_{n}\left(\varpi_{1}, \varpi_{2}\right)\right)\left[\begin{array}{ll}
I & 0
\end{array}\right]\right)\right)
$$

According to the generalized KYP lemma (Proposition 1), the dilated error system $\mathscr{E}_{n}(J \omega)$ thus satisfies:

$$
\sigma_{\max }\left(\mathscr{E}_{n}(J \omega)\right) \leq \sqrt{\eta_{n}\left(\varpi_{1}, \varpi_{2}\right)} \text { for all } \omega \in\left[\varpi_{1}, \varpi_{2}\right] .
$$

Therefore, the error system satisfies the following inequality:

$$
\sigma_{\max }\left(\mathcal{E}_{n}(J \omega)\right) \leq \sigma_{\max }\left(\mathscr{E}_{n}(J \omega)\right) \leq \sqrt{\eta_{n}\left(\varpi_{1}, \varpi_{2}\right)} \quad \text { for all } \quad \omega \in\left[\varpi_{1}, \varpi_{2}\right]
$$

This completes the proof of the interval-type error bound (25) for $r=n-1$, the cases $r=n-2, \ldots, 1$ follow step by step.

(c) The proof of the EF-type error bound (31) for interval-type FDBT can be completed in full analogy to the proof of the EF-type error bound (18) for SF-type FDBT.

Similar to the SF-type balanced realization case, we can also study the limiting behavior of the interval-type frequency-dependent Gramians.

Proposition 5 Assuming that the Lyapunov equations (11) corresponding to the original system (1) has unique solutions and a balanced realization of (1) exists. Then, the interval-type frequency-dependent Gramians have the following properties:

1. $\lim _{\varpi_{d} \rightarrow 0} W_{c}\left(\varpi_{1}, \varpi_{2}\right)=0, \lim _{\varpi_{d} \rightarrow 0} W_{o}\left(\varpi_{1}, \varpi_{2}\right)=0$,

$\lim _{\varpi_{d} \rightarrow 0} \Sigma_{\varpi}\left(\varpi_{1}, \varpi_{2}\right)=0$.

2. $\lim _{\varpi_{d} \rightarrow \infty} W_{c}\left(\varpi_{1}, \varpi_{2}\right)=W_{c}, \lim _{\varpi_{d} \rightarrow \infty} W_{o}\left(\varpi_{1}, \varpi_{2}\right)=W_{o}$, $\lim _{\varpi_{d} \rightarrow \infty} \Sigma_{\varpi}\left(\varpi_{1}, \varpi_{2}\right)=\Sigma$.

3. $\lim _{\varpi_{d} \rightarrow 0} \eta_{i}\left(\varpi_{1}, \varpi_{2}\right)=0$ for $i=1, \ldots, n$.

Proof 1. It can be easily observed that:

$$
\begin{aligned}
\lim _{\varpi_{d} \rightarrow 0} A\left(\varpi_{1}, \varpi_{2}\right) & =\lim _{\varpi_{d} \rightarrow 0} A=A, \\
\lim _{\varpi_{d} \rightarrow 0} B\left(\varpi_{1}, \varpi_{2}\right) & =\lim _{\varpi_{d} \rightarrow 0}\left(\varpi_{d}^{2}\left(\jmath \varpi_{1} I-A\right)^{-1}\left(\jmath \varpi_{2} I-A\right)^{-1}\right)^{\frac{1}{2} \star} B=0, \\
\lim _{\varpi_{d} \rightarrow 0} C\left(\varpi_{1}, \varpi_{2}\right) & =\lim _{\varpi_{d} \rightarrow 0} C\left(\varpi_{d}^{2}\left(j \varpi_{1} I-A\right)^{-1}\left(j \varpi_{2} I-A\right)^{-1}\right)^{\frac{1}{2} \star}=0 .
\end{aligned}
$$

From the interval-type frequency-dependent Lyapunov (22), we thus get:

$$
\begin{aligned}
& A \lim _{\varpi_{d} \rightarrow 0} W_{c}\left(\varpi_{1}, \varpi_{2}\right)+\lim _{\varpi_{d} \rightarrow 0} W_{c}\left(\varpi_{1}, \varpi_{2}\right) A^{*}=0, \\
& A^{*} \lim _{\varpi_{d} \rightarrow 0} W_{o}\left(\varpi_{1}, \varpi_{2}\right)+\lim _{\varpi_{d} \rightarrow 0} W_{o}\left(\varpi_{1}, \varpi_{2}\right) A=0 .
\end{aligned}
$$

The unique solvability of the Lyapunov equations (11) implies that the Lyapunov operators $X \rightarrow A X+X A^{*}$ as well as $Y \rightarrow A^{*} Y+Y A$ have trivial 
kernels. This implies $\lim _{\varpi_{d} \rightarrow 0} W_{c}\left(\varpi_{1}, \varpi_{2}\right)=0$ and $\lim _{\varpi_{d} \rightarrow 0} W_{o}\left(\varpi_{1}, \varpi_{2}\right)=$ 0 . The result for $\Sigma_{\varpi}\left(\varpi_{1}, \varpi_{2}\right)$ follows using the balanced realization.

2. Similar to the proof of part 1, we have:

$$
\begin{aligned}
& \lim _{\varpi_{d} \rightarrow \infty} A\left(\varpi_{1}, \varpi_{2}\right)=\lim _{\varpi_{d} \rightarrow \infty} A=A, \\
& \lim _{\varpi_{d} \rightarrow \infty} B\left(\varpi_{1}, \varpi_{2}\right)=\lim _{\varpi_{d} \rightarrow \infty}\left(\varpi_{d}^{2}\left(\jmath \varpi_{1} I-A\right)^{-1}\left(\jmath \varpi_{2} I-A\right)^{-1}\right)^{\frac{1}{2} \star} B=B, \\
& \lim _{\varpi_{d} \rightarrow \infty} C\left(\varpi_{1}, \varpi_{2}\right)=\lim _{\varpi_{d} \rightarrow \infty} C\left(\varpi_{d}^{2}\left(\jmath \varpi_{1} I-A\right)^{-1}\left(\jmath \varpi_{2} I-A\right)^{-1}\right)^{\frac{1}{2} \star}=C .
\end{aligned}
$$

and

$$
\begin{array}{r}
A \lim _{\varpi_{d} \rightarrow \infty} W_{c}\left(\varpi_{1}, \varpi_{2}\right)+\lim _{\varpi_{d} \rightarrow \infty} W_{c}\left(\varpi_{1}, \varpi_{2}\right) A^{*}+B B^{*}=0, \\
A^{*} \lim _{\varpi_{d} \rightarrow \infty} W_{o}\left(\varpi_{1}, \varpi_{2}\right)+\lim _{\varpi_{d} \rightarrow \infty} W_{o}\left(\varpi_{1}, \varpi_{2}\right) A+C^{*} C=0 .
\end{array}
$$

Thus, again employing the unique solvability of the Lyapunov (11), we can conclude:

$$
\lim _{\varpi_{d} \rightarrow \infty} W_{c}\left(\varpi_{1}, \varpi_{2}\right)=W_{c} \quad \text { and } \quad \lim _{\varpi_{d} \rightarrow \infty} W_{o}\left(\varpi_{1}, \varpi_{2}\right)=W_{o} .
$$

The result for $\Sigma_{\varpi}\left(\varpi_{1}, \varpi_{2}\right)$ follows using the balanced realization.

3. As $\sigma_{i}\left(\varpi_{1}, \varpi_{2}\right)$ are the diagonal entries of $\Sigma_{\varpi}\left(\varpi_{1}, \varpi_{2}\right)$, it follows from part 1 that

$$
\lim _{\varpi_{d} \rightarrow 0} \sigma_{i}\left(\varpi_{1}, \varpi_{2}\right)=0 .
$$

With this and the definition of $\eta_{i}\left(\varpi_{1}, \varpi_{2}\right)$ as well as the boundedness of $\mathscr{C}_{e i}$, $\mathcal{N}_{e i}$ and $\mathscr{B}_{e i}$ as $\varpi_{d} \rightarrow 0$, part 3 follows.

Finally, we need a simple identity to derive the interval-type FDBT method. The following lemma directly follows from the definition of matrix functions.

\section{Lemma 1 The equation}

$$
\begin{aligned}
& T^{-1}\left[\varpi_{d}^{2}\left(\jmath \varpi_{1} I-A\right)^{-1}\left(j \varpi_{2} I-A\right)^{-1}\right]^{\frac{1}{2} \star} T \\
& =\left(\varpi_{d}^{2}\left(j \varpi_{1} I-T^{-1} A T\right)^{-1}\left(j \varpi_{2} I-T^{-1} A T\right)^{-1}\right)^{\frac{1}{2} \star}
\end{aligned}
$$

holds for any invertible matrix $T \in \mathbb{C}^{n \times n}$.

With these preparations, the interval-type FDBT algorithm is stated as Algorithm 2.

Remark 6 Compared with other balancing-related approaches, the most distinctive feature of the proposed interval-type FDBT method is that it gives an interval-type 


\section{Algorithm 2 Interval-type FDBT.}

Input: Full-order model $(A, B, C, D)$, frequency interval $\left(\varpi_{1}, \varpi_{2}\right)$ with $0 \leq \varpi_{1}<$ $\varpi_{2}$, order of $r$ of the ROM.

Output: ROM $\left(A_{r}, B_{r}, C_{r}, D_{r}\right)$.

Step 1. Solve the interval-type frequency-dependent Lyapunov (22)

Step 2. Get the frequency-dependent realization of the given system by coordinate transformation:

$$
\left[\begin{array}{c|c}
A_{b} & B_{b} \\
\hline C_{b} & D_{b}
\end{array}\right]=\left[\begin{array}{c|c}
T^{-1}\left(\varpi_{1}, \varpi_{2}\right) A T\left(\varpi_{1}, \varpi_{2}\right) & T^{-1}\left(\varpi_{1}, \varpi_{2}\right) B \\
\hline C T\left(\varpi_{1}, \varpi_{2}\right) & D+C_{b}\left[\left(J \varpi_{c} I-A_{b}\right)\left(J \varpi_{1} I-A_{b}\right)^{-1}\left(J \varpi_{2} I-A_{b}\right)^{-1}\right] B_{b}
\end{array}\right],
$$

where $T\left(\varpi_{1}, \varpi_{2}\right)$ is a matrix that simultaneously diagonalizes the matrices $W_{c}\left(\varpi_{1}, \varpi_{2}\right)$ and $W_{o}\left(\varpi_{1}, \varpi_{2}\right)$, i.e.,

$T^{-1}\left(\varpi_{1}, \varpi_{2}\right) W_{c}\left(\varpi_{1}, \varpi_{2}\right) T\left(\varpi_{1}, \varpi_{2}\right)=T^{*}\left(\varpi_{1}, \varpi_{2}\right) W_{o}\left(\varpi_{1}, \varpi_{2}\right) T^{-*}\left(\varpi_{1}, \varpi_{2}\right)=\Sigma\left(\varpi_{1}, \varpi_{2}\right)$,

Step 3. Compute the reduced-order model as:

$$
\begin{aligned}
& A_{r}=Z_{r} A_{b} Z_{r}^{T}, \\
& B_{r}=\left[\varpi_{d}^{2}\left(\jmath \varpi_{1} I-A_{r}\right)^{-1}\left(j \varpi_{2} I-A_{r}\right)^{-1}\right]^{-\frac{1}{2} \star} Z_{r}\left[\varpi_{d}^{2}\left(\jmath \varpi_{1} I-A_{b}\right)^{-1}\left(j \varpi_{2} I-A_{b}\right)^{-1}\right]^{\frac{1}{2} \star} B_{b}, \\
& C_{r}=C_{b}\left[\varpi_{d}^{2}\left(j \varpi_{1} I-A_{b}\right)^{-1}\left(j \varpi_{2} I-A_{b}\right)^{-1}\right]^{\frac{1}{2} \star} Z_{r}^{T}\left[\varpi_{d}^{2}\left(j \varpi_{1} I-A_{r}\right)^{-1}\left(J \varpi_{2} I-A_{r}\right)^{-1}\right]^{-\frac{1}{2} \star} \text {, } \\
& D_{r}=D+C_{b}\left[\left(\jmath \varpi_{c} I-A_{b}\right)\left(\jmath \varpi_{1} I-A_{b}\right)^{-1}\left(\jmath \varpi_{2} I-A_{b}\right)^{-1}\right] B_{b} \\
& -C_{r}\left[\left(\jmath \varpi_{c} I-A_{r}\right)\left(\mathrm{J} \varpi_{1} I-A_{r}\right)^{-1}\left(\jmath \varpi_{2} I-A_{r}\right)^{-1}\right] B_{r} .
\end{aligned}
$$

error bound (25). To the best of our knowledge, it is the first time such an intervaltype error bound is provided using the interval-type index (4). In particular, as revealed by Proposition 5, the interval-type error bound (25) always tends to zero when the interval size tends to zero. This property means that the interval-type FDBT generally will give rise to good in-band approximation performance as long as the size of frequency interval is small enough. On the other hand, the interval-type error bound may be increasing quickly with respect to the size of the frequency interval. So, there is still need for improvement in order to have a proven small error in larger frequency intervals.

Remark 7 The interval-type FDBT is presented in a rather general form, i.e., the system matrices are allowed to be complex or real and the frequency interval might be symmetric or non-symmetric w.r.t. the origin. It can be easily verified that the interval-type FDBT will generate real reduced models for real full models if the given frequency interval is symmetric (i.e., $\varpi_{1}=-\varpi_{2}$ ). For applications with real sys- 
tem parameter restriction in asymmetric frequency interval cases $\left(\omega \in\left[\varpi_{1}, \varpi_{2}\right]\right)$, the interval-type FDBT can also be applied in a conservative way by modifying the frequency as $\omega \in\left[-\varpi_{\max }, \varpi_{\max }\right]$ with $\varpi_{\max }=\max \left\{\left|\varpi_{1}\right|,\left|\varpi_{2}\right|\right\}$.

\section{Numerical examples}

Example 1 Consider an LTI system (1) defined by the following data:

$$
\left[\begin{array}{l|l}
A \mid B \\
\hline C & D
\end{array}\right]=\left[\begin{array}{rrrrrr|r}
0.2128 & 0.7749 & 0.1945 & -0.2864 & 0.0501 & -0.0464 & 0.9673 \\
-0.6613 & -2.6801 & -0.8468 & -0.5733 & -0.7945 & 0.9653 & -1.4467 \\
0.2423 & -0.8043 & -0.7669 & -0.5423 & -0.9032 & 0.1441 & -1.2514 \\
-0.1508 & 0.5229 & 0.6927 & -0.0704 & 0.8778 & -0.5350 & -0.4141 \\
0.3542 & 0.7882 & 0.3681 & -0.2077 & -0.1705 & -0.7660 & -0.6560 \\
-0.6424 & -0.5045 & -0.0252 & 0.6453 & 0.9838 & -0.9392 & -0.1651 \\
\hline-1.5883 & -1.3181 & 0.5656 & 1.1507 & -0.5106 & -0.7736 & 3.9764
\end{array}\right]
$$

Here, we assume that the frequency of the input signal belongs to an uncertain interval around $\varpi=0$. The task is to build a ROM of order 3 approximating the frequency domain dynamic behavior of the original model well in the neighborhood of $\varpi=0$. Among the existing balancing-related methods, the (generalized) SPA is the most suitable one for coping with this kind of MOR problem. At the same time, our proposed SF-type FDBT method can also be applied for this kind of problems. The sigma plots of the error systems generated by generalized SPA and SF-type FDBT are depicted in Figs. 1 and 2, respectively. As these figures show, both of them can give rise to a small approximation error around $\varpi=0$. Moreover, one can make a tradeoff between the local approximation performance and global approximation performance by adjusting the user-defined parameter ( $\rho$ for generalized SPA and $\epsilon$ for SF-type FDBT). In this example, the generalized SPA and the SF-type FDBT perform very similarly. However, a huge variety in their performance may occur in some cases; see, e.g., Example 3 below, in which only the SF-type FDBT is effective.

Besides, the corresponding SF-type error bound and EF-type error bound with respect to different values of $\epsilon$ provided by SF-type FDBT are plotted in Fig. 3. According to the error bounds, we know that the local and global approximation performance could be well balanced by picking the value of parameter $\epsilon$ larger than 3 and smaller than 5. In this way, the trial-and-error procedure to find an appropriate $\epsilon$ can be shortened or avoided. Furthermore, if the parameter $\epsilon$ satisfies $25>\epsilon>4$, the EF-type error bound of SF-type FDBT will even be smaller than the EF-type error bound of FIBT. 


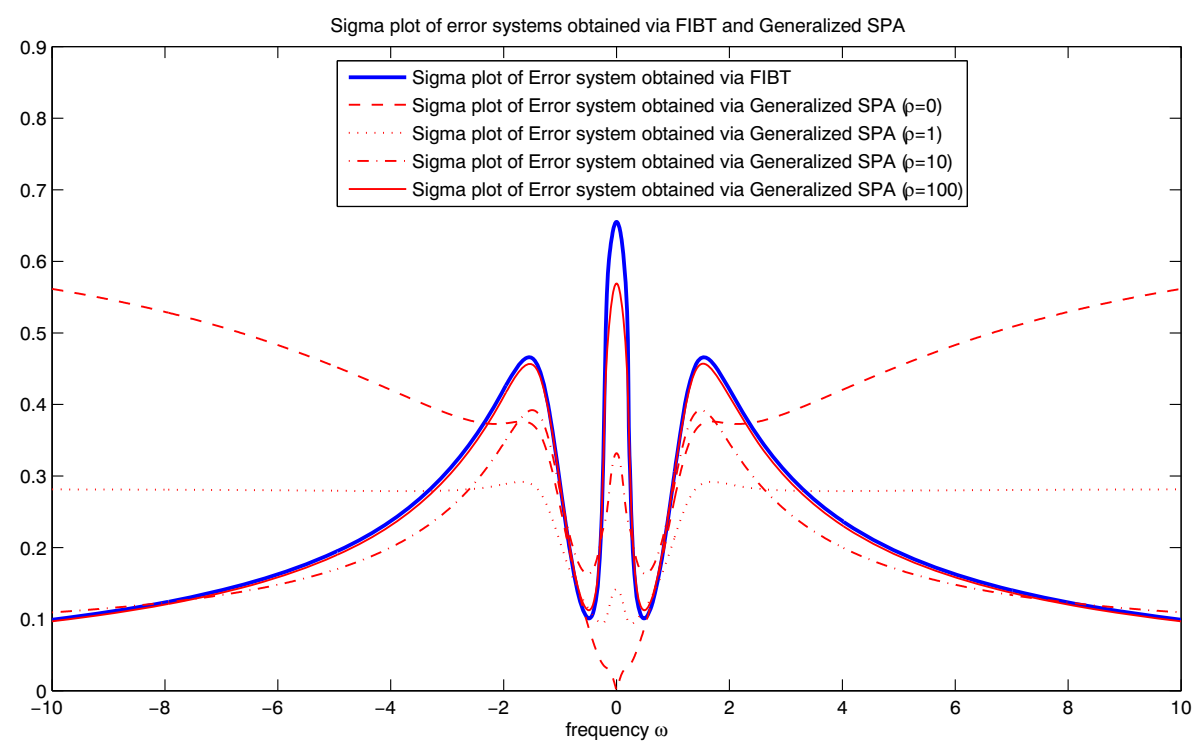

Fig. 1 Sigma plot of error systems generated via generalized SPA and FIBT

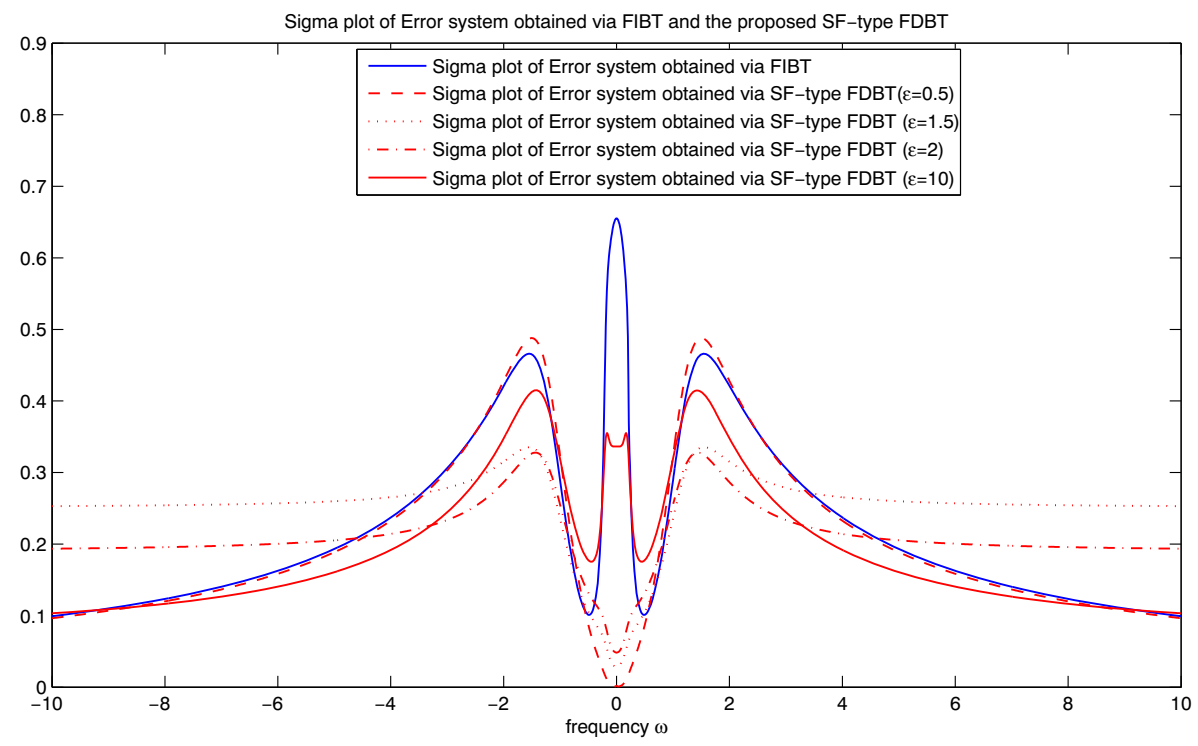

Fig. 2 Sigma plot of error systems generated via SF-type FDBT and FIBT 


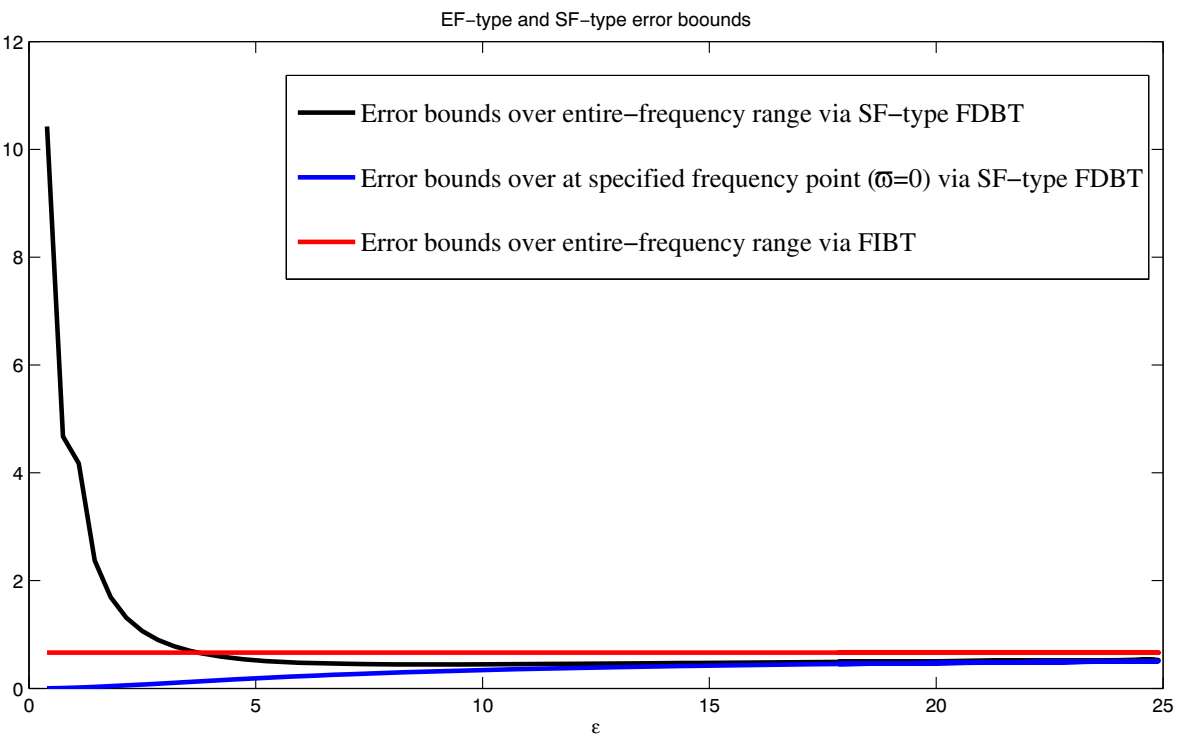

Fig. 3 SF-type error bound and EF-type error bound with respect to the parameter $\epsilon$

Example 2 Now, consider an LTI system (1) given via

$$
\left[\begin{array}{l|l}
A & B \\
\hline C & D
\end{array}\right]=\left[\begin{array}{rrrr|r}
-0.62 & 0.44 & -0.03 & -0.00 & -0.31 \\
0.44 & -3.64 & 0.59 & 0.02 & 0.47 \\
0.03 & -0.59 & -6.80 & -0.46 & 0.12 \\
-0.00 & 0.02 & 0.46 & -5.64 & -0.00 \\
\hline-0.31 & 0.47 & -0.12 & -0.00 & 0.00
\end{array}\right]
$$

Here, the frequency range of the input signals is assumed to be pre-known, and we consider the following two different cases:

Case 1: $\quad \omega \in[-0.4,+0.4]$;

Case 2: $\omega \in[-0.8,+0.8]$.

Among the existing balancing-related methods, FGBT [17] is the one developed for solving such interval-type finite-frequency MOR problems. Our proposed intervaltype FDBT also aims at solving this kind of problems. We will show the differences between them by this example. The sigma plot of the error systems and the corresponding error bound are given in Figs. 4 and 5. A striking difference on the type of error bounds can be observed in these plots. FGBT provides an error bound for the entire-frequency range; in contrast, the interval-type FDBT procedure yields an error bound for the pre-specified frequency interval. Since it is assumed that the operating frequencies belong to the given intervals, the interval-type error bounds are adequate for approximation of the true errors. Compared with the standard FIBT, both the FGBT and the interval-type FDBT methods are effective in improving the 


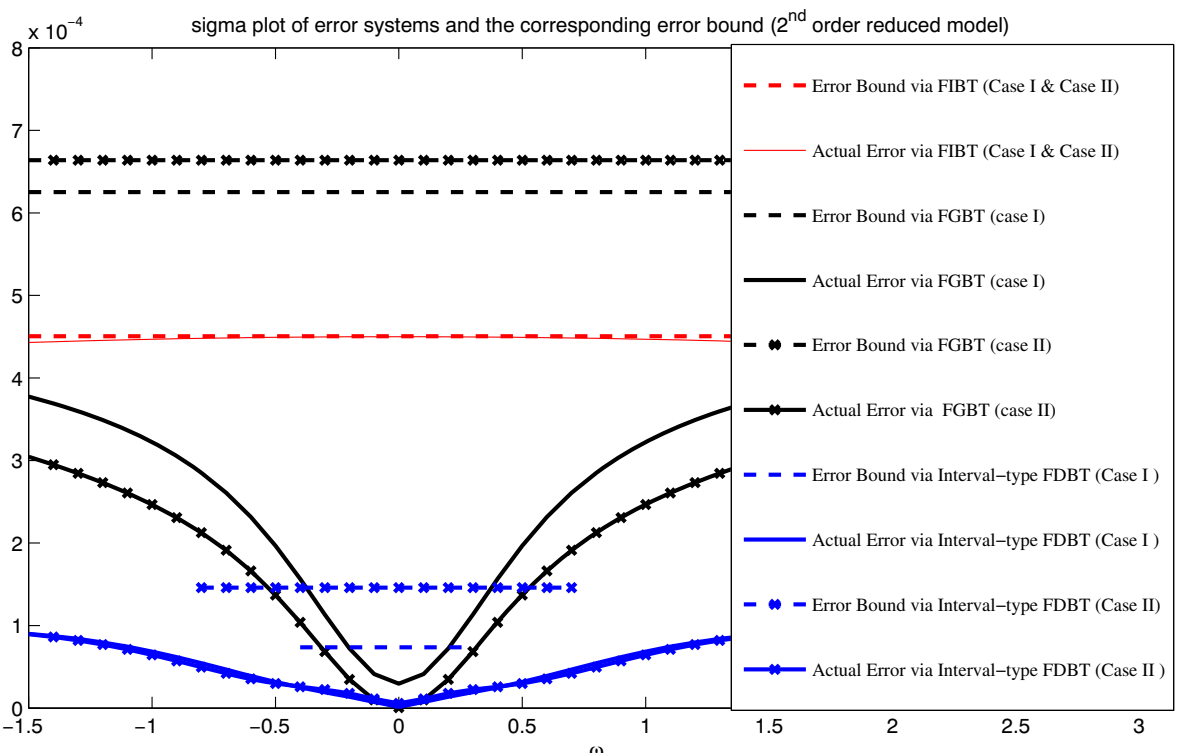

Fig. 4 Sigma plot of error models and the corresponding error bounds ( $2^{\text {nd }}$-order ROMs)

approximation performance in the specified frequency interval. At the same time, the interval-type FDBT has the advantage that it gives rise to better approximation performance and smaller error bound simultaneously.

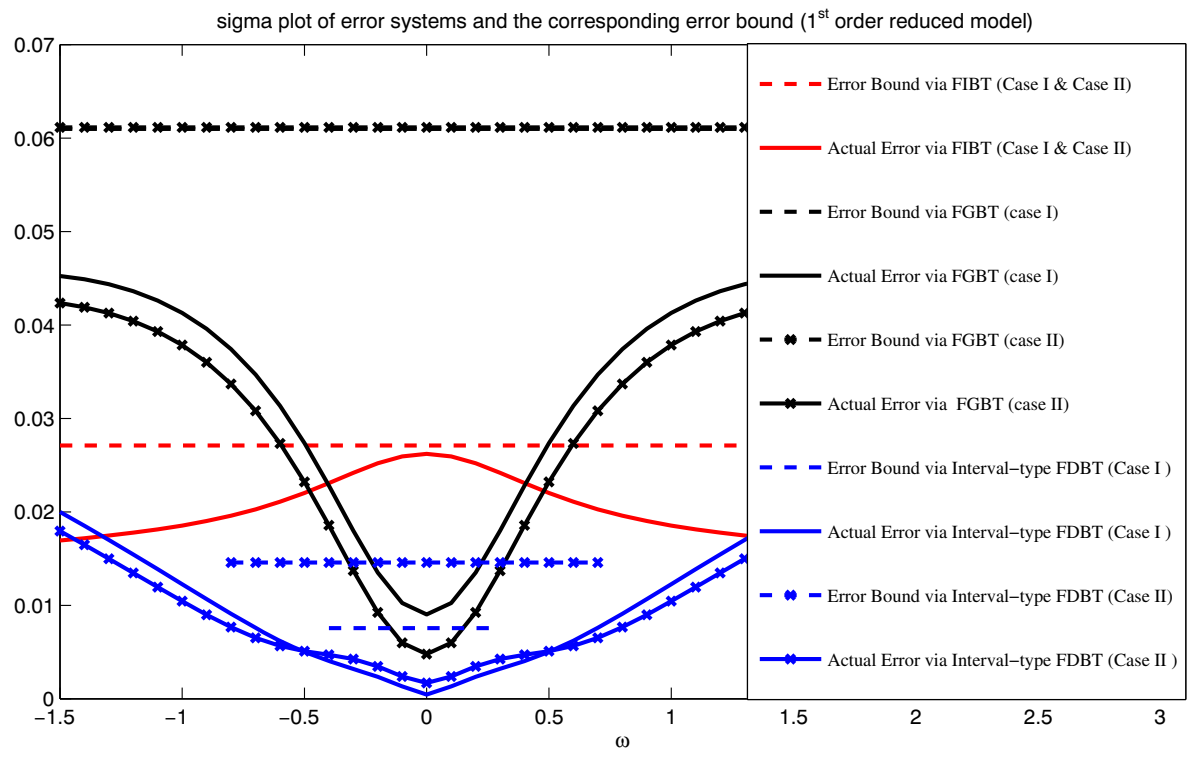

Fig. 5 Sigma plot of error systems and the corresponding error bounds ( $1^{\text {st }}$-order ROMs) 
As depicted by Figs. 4 and 5, the interval-type error bound provided by intervaltype FDBT for case 2 is larger than the interval-type error bound for case 1 . To further show the properties of the interval-type error bound, we plot the curves of the two interval size $\left(\varpi_{l}\right)$-dependent indices in Figs. 6 and 7. As expected, it can be observed that the interval-type error bound appears to be increasing with respect to the interval size. Moreover, the interval-type FDBT outperforms FGBT and FIBT w.r.t. both the in-band approximation performance and the error bound for the cases that $\varpi_{l}<1.5$.

As discussed in Remark 6, the interval-type FDBT always provides a small error bound as long as the size of the frequency interval is small enough. To show this, a randomization experiment was carried out. We randomly generate 100 stable systems of order 4. (The off-diagonal elements of matrix $A$ and each element of matrices $B, C, D$ are obtained with a zero mean and unitary variance normal distribution, the diagonal element of matrix $A$ are obtained with mean -5.5 and variance 4.5.) To compare the average performance between FGBT and interval-type FDBT, several indices are defined in Table 2.

In Table 2, $\varpi_{l}$ represents the upper bound of the symmetric frequency interval, $r$ is the order of the ROM, and $G_{D r}^{l}(J \omega), G_{S r}^{l}(J \omega), G_{G r}^{l}(\jmath \omega)$, and $G_{I r}^{l}(J \omega)$ represent the ROMs of order $r$ generated by interval-type FDBT, SPA, FGBT, and the classic FIBT for the $l^{\text {th }}$ random model, respectively. Figures 8 and 9 display the experimental results for these error indices.

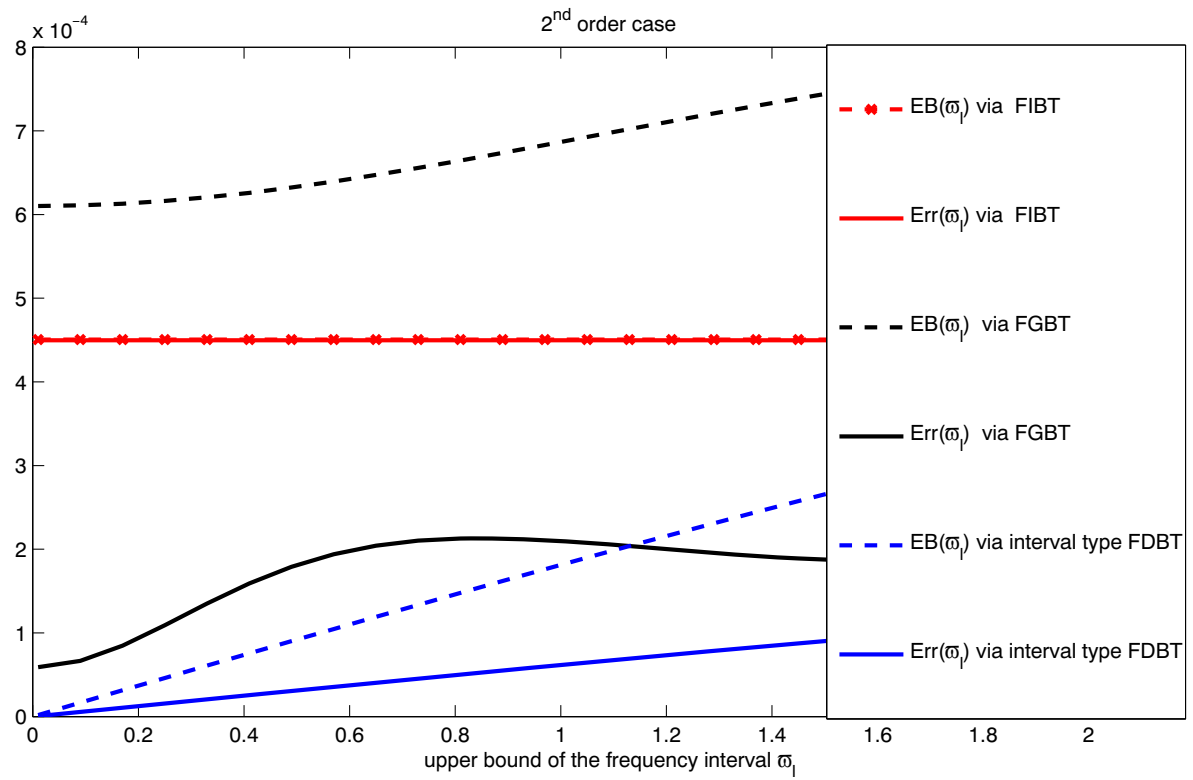

Fig. 6 Curves of maximum error and error bound ( $1^{s t}$-order ROM). $\operatorname{Err}\left(\varpi_{l}\right)$ represents the maximum approximation error for the frequency interval $\left[-\varpi_{l}, \varpi_{l}\right]$, i.e., $\operatorname{Err}\left(\varpi_{l}\right)=\sigma_{\max }\left(G(j \omega)-G_{r}(j \omega)\right), \forall \omega \in$ $\left[-\varpi_{l}, \varpi_{1}\right]$, where $G_{r}(j \omega)$ denotes the ROM generated by the specified method. $\operatorname{Err}\left(\varpi_{l}\right)$ represents the interval-type error bound for interval-type FDBT 


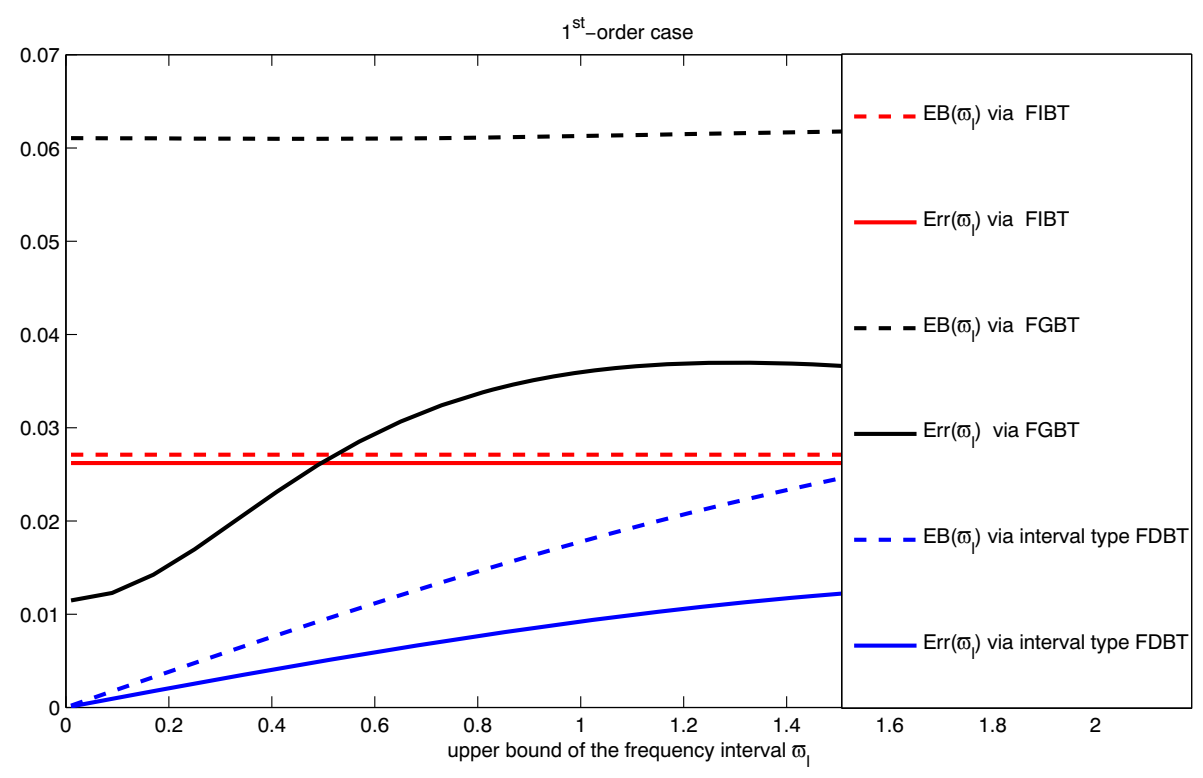

Fig. 7 Curves of maximum error and error bound $\left(2^{\text {nd }}\right.$-order ROM). $\operatorname{Err}\left(\varpi_{l}\right)$ represents the maximum approximation error for the frequency interval $\left[-\varpi_{l}, \varpi_{l}\right]$, i.e., $\operatorname{Err}\left(\varpi_{l}\right)=\sigma_{\max }\left(G(j \omega)-G_{r}(j \omega)\right), \forall \omega \in$ $\left[-\varpi_{l}, \varpi_{1}\right]$, where $G_{r}(j \omega)$ denotes the ROM generated by the specified method. $\operatorname{Err}\left(\varpi_{l}\right)$ represents the interval-type error bound for interval-type FDBT

Figure 8 validates that the interval-type error bound provided by interval-type FDBT generally is smaller than the EF-type error bound generated by FIBT and FDBT for the cases that the interval size is small enough (about $\varpi<1$ in this experiment). Although the advantage w.r.t. the error bound is restricted for small interval-size cases, it is suggested to take the interval-type FDBT as a feasible option even for medium interval-size cases. According to our experiment, the interval-type FDBT generally also gives rise to better in-band approximation performance than FIBT and FGBT for medium interval-size cases (see Fig. 9 for an illustration).

Table 2 Indices used to compare the approximation error and error bound generated by different methods

Indexes

$\operatorname{Err}\left(\varpi_{l}, r, \mathrm{FDBT}\right)$

$\operatorname{Err}\left(\varpi_{l}, r, \mathrm{FGBT}\right)$

$\mathrm{Eb}\left(\varpi_{l}, r, \mathrm{FDBT}\right)$

$\mathrm{Eb}\left(\varpi_{l}, r, \mathrm{FGBT}\right)$
Computation formula

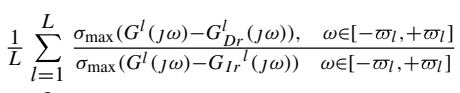

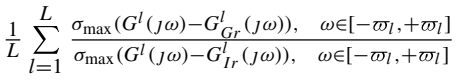

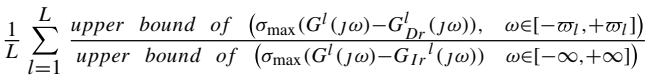

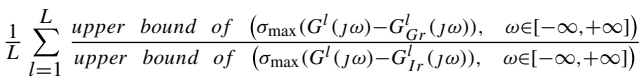




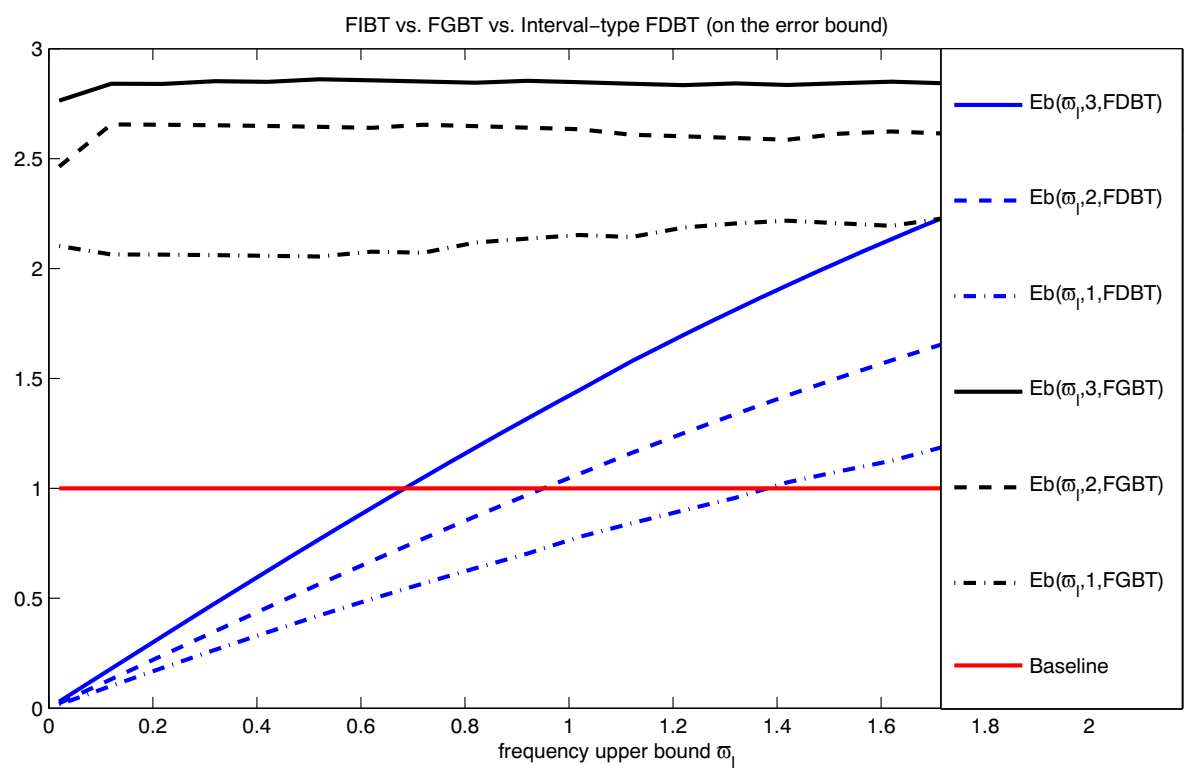

Fig. 8 Randomized experiment results on actual error

Example 3 Consider the $201^{\text {st }}$-order RLC ladder circuit example provided in [17]. As pointed out in [17], approximating the ladder circuit is quite difficult in the framework of balancing related model order reduction approaches since neither the Hankel

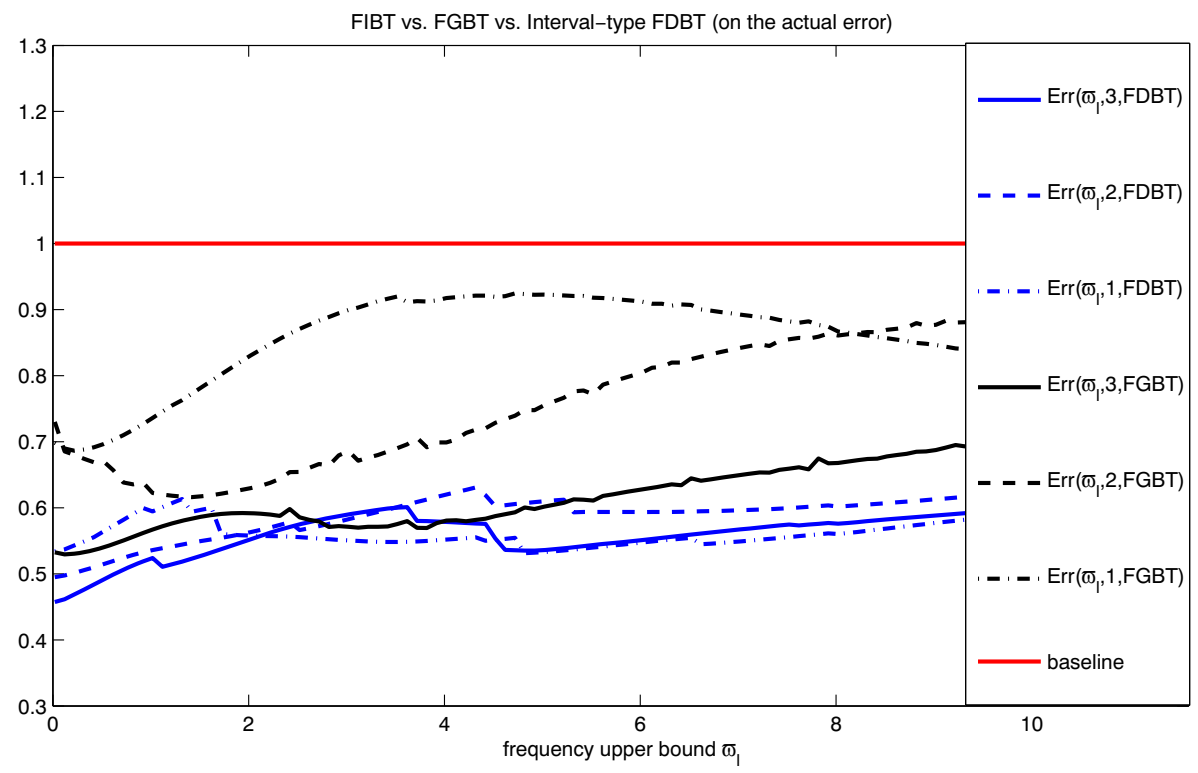

Fig. 9 Randomized experiment results on error bound 
nor the singular values decay to any extent. In particular, its dynamic behavior over low frequency ranges is too complex to be well approximated due to the special distribution of its poles and zeros. Here, we are interested to approximate this circuit in the following cases:

Case 1: The frequency of the input signal belongs to an unknown neighborhood of dominating operating frequency point $(\varpi=0)$.

Case 2: The frequency of the input signal is known to be within the interval $(\omega \in$ $[-0.5,+0.5])$.

First, let us consider case 1 and apply FIBT and generalized SPA to build ROMs. The frequency response of the full-order model and the ROM of order 181 are shown in Fig. 10, i.e., only 20 states are removed here from the system.

As indicated by the visual inspections of the frequency response of the reduced vs. the full system from Fig. 10, the standard FIBT fails to approximate the dynamic behaviors around $\omega=0$ even when the order of the ROM is 181. Besides, it is surprising and remarkable that the generalized SPA method also fails here. Although the generalized SPA approach generally leads to good approximation performance around $\omega=0$, it is incapable to cope with this example. Now, let us resort to the proposed SF-type FDBT for dealing with the MOR problem in case 1. Our experimental results show that good approximants can be generated via SF-type FDBT as long as the order of the ROM is larger than 50. The frequency responses of the full and reduced systems shown in Fig. 11 show a success of SF-type FDBT for this example. In addition, the frequency response of a ROM generated by Padé approximation (i.e., moment-matching at zero) is also included in Fig. 11. It can be seen that Padé

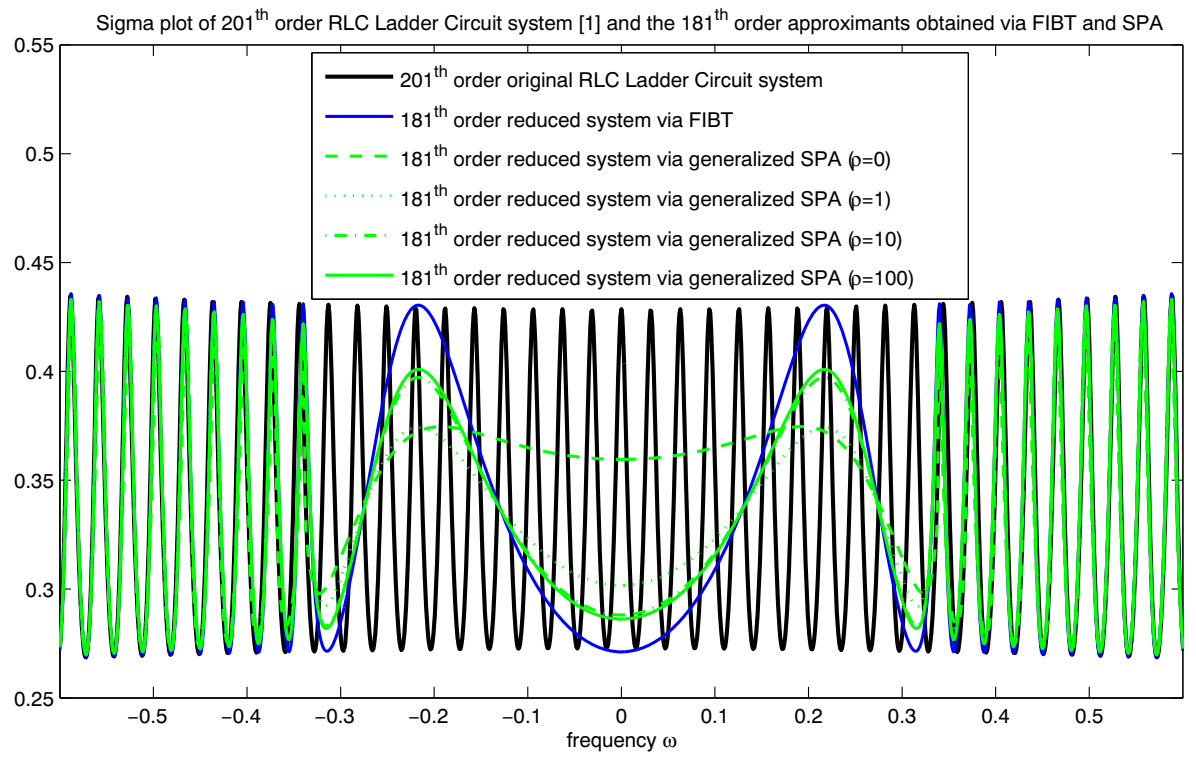

Fig. 10 Approximating the ladder circuit in Case I via FIBT \& Generalized SPA 


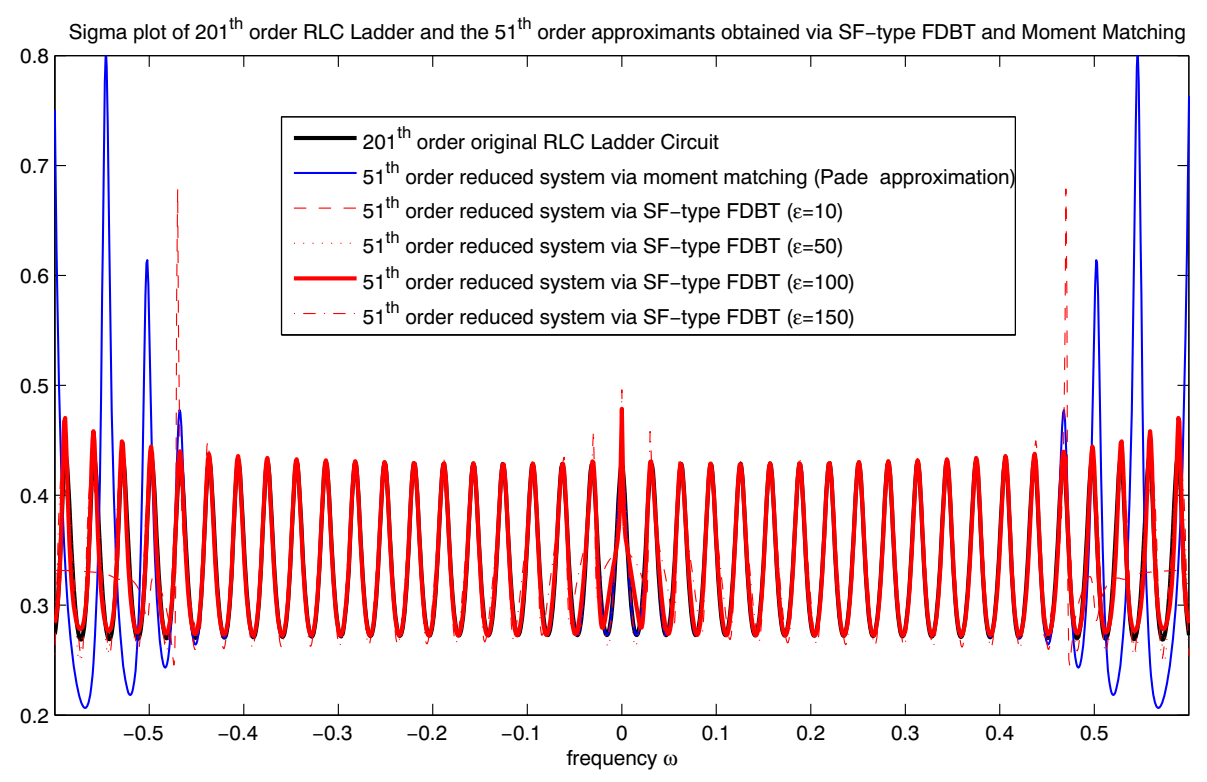

Fig. 11 Approximating the ladder circuit in case I via SF-type FDBT and Moment matching

approximation also leads to good approximation performance, which is both natural and expected since it is an inherent local approximation method. It is interesting that the performance of interval-type FDBT is very similar to Padé approximation for this

Sigma plot of $201^{\text {th }}$ order RLC Ladder Circuit system, $61^{\text {th }}$ order and $51^{\text {th }}$ order approximants obtained via Interval-type FDBT and FGBT

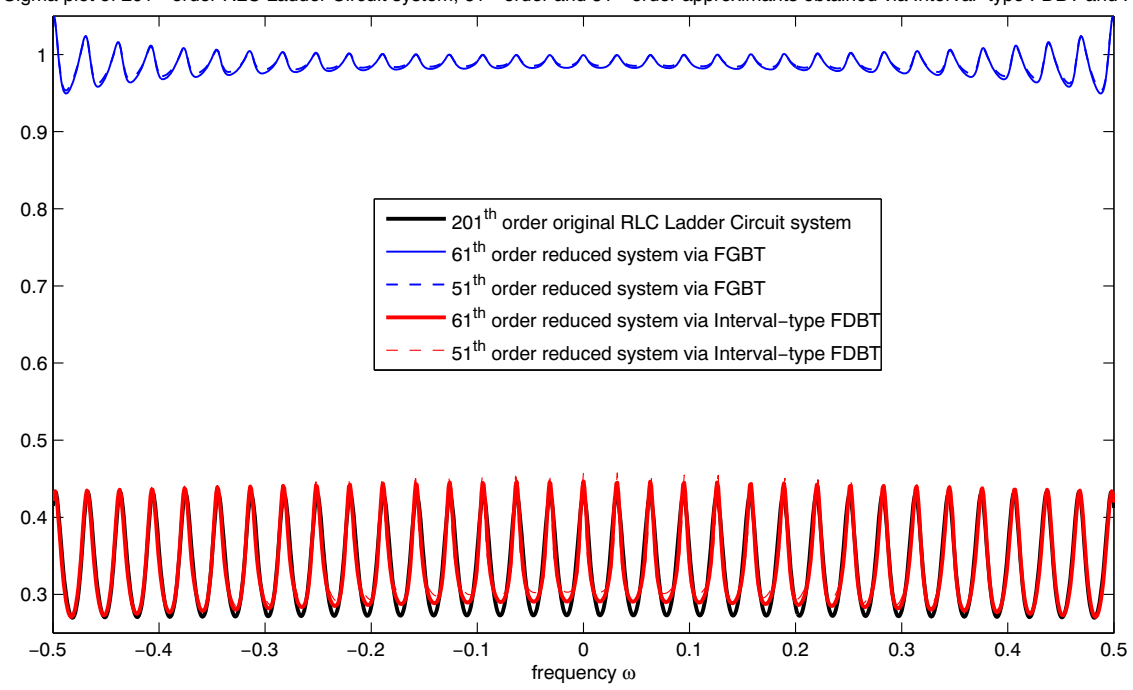

Fig. 12 Approximating the ladder circuit in case II via interval-type FDBT and FGBT 
example. This provides an example that good local approximation performance of the ladder circuit may also be obtained in the balancing-related framework.

Finally, consider the stated MOR problem in case 2 and apply the interval-type FDBT and FGBT [17] to build ROMs. Figure 12 shows the frequency response of the full-order model and ROMs of order 61 and 51. The results show that only the interval-type FDBT leads to satisfactory in-band approximation performance.

\section{Conclusions and future work}

This paper revisited model order reduction over limited frequency intervals in the framework of balanced truncation. We have developed SF-type and interval-type frequency-dependent balanced truncation methods to cope with the partially preknown frequency interval cases and the completely pre-known frequency interval cases, respectively. Moreover, for the first time, SF-type and interval-type error bounds have been established that assess the error only in the desired frequency band and are thus sharper than previous bounds that use the entire frequency range. Numerical examples illustrate the efficiency and advantages of the proposed methods. Future work will focus on developing frequency-dependent balanced truncation algorithms in other forms to get even sharper frequency-dependent error bounds.

Funding Open Access funding enabled and organized by Projekt DEAL.

Open Access This article is licensed under a Creative Commons Attribution 4.0 International License, which permits use, sharing, adaptation, distribution and reproduction in any medium or format, as long as you give appropriate credit to the original author(s) and the source, provide a link to the Creative Commons licence, and indicate if changes were made. The images or other third party material in this article are included in the article's Creative Commons licence, unless indicated otherwise in a credit line to the material. If material is not included in the article's Creative Commons licence and your intended use is not permitted by statutory regulation or exceeds the permitted use, you will need to obtain permission directly from the copyright holder. To view a copy of this licence, visit http://creativecommonshorg/licenses/by/4. $0 \%$.

\section{References}

1. Antoulas, A.C.: Approximation of large-scale dynamical systems, SIAM Publications, Philadelphia (2005)

2. Baur, U., Benner, P., Feng, L.: Model order reduction for linear and nonlinear systems: A systemtheoretic perspective. Arch. Comp. Eng. 21(4), 331-358 (2014). https://doi.org/10.1007/s11831014-9111-2

3. Benner, P., Cohen, A., Ohlberger, M., Willcox, K.: Model reduction and approximation: Theory and algorithms. SIAM, Philadelphia, PA (2017)

4. Benner, P., Hinze, M., ter Maten, E.JW.: Model reduction for circuit simulation. Lecture Notes in Electrical Engineering, vol. 74. Springer, New York (2011)

5. Benner, P., Mehrmann, V., Sorensen, D.C.: Dimension reduction of large-scale systems. Lecture Notes in Computational Sciences and Engineering, vol. 45. Springer, Berlin (2005)

6. Hesthaven, J.S., Rozza, G., Stamm, B.: Certified reduced basis methods for parametrized partial differential equations. SpringerBriefs in Mathematics. Springer, Berlin (2016) 
7. Quarteroni, A., Manzoni, A., Negri, F.: Reduced basis methods for partial differential equations. La Matematica per il 3+2, vol. 92. Springer, Berlin (2016)

8. Schilders, W.HA., van der Vorst, H.A., Rommes, J.: Model order reduction: Theory, research aspects and applications. Springe, Berlin (2008)

9. Obinata, G., Anderson, B.DO.: Model reduction for control system design. Communications and Control Engineering Series. Springer, London (2001)

10. Moore, B.C.: Principal component analysis in linear systems: controllability, observability, and model reduction. IEEE Trans. AC AC-26(1), 17-32 (1981)

11. Anderson, B.DO., Liu, Y.: Controller reduction: concepts and approaches. IEEE Trans. AC 34(8), 802-812 (1989)

12. Benner, P., Kürschner, P., Saak, J.: Frequency-limited balanced truncation with low-rank approximations. SIAM Sci. Comp. 38(1), A471-A499 (2016)

13. Enns, D.F.: Model reduction with balanced realizations: An error bound and a frequency weighted generalization. In: Proc. 23rd IEEE Conf. Decision Contr., vol. 23, pp. 127-132 (1984)

14. Gawronski, W., Juang, J.: Model reduction in limited time and frequency intervals. Int. J. Syst. Sci. 21(2), 349-376 (1990). https://doi.org/10.1080/00207729008910366

15. Ghafoor, A., Sreeram, V.: Model reduction via limited frequency interval gramians. IEEE Trans. Circ. Syst. I 55(9), 2806-2812 (2008)

16. Ghafoor, A., Sreeram, V.: A survey/review of frequency-weighted balanced model reduction techniques. ASME J. Dyn. Syst. Meas. Cont. 130, 061004-1-061004-16 (2008)

17. Gugercin, S., Antoulas, A.C.: A survey of model reduction by balanced truncation and some new results. Int. Control 77(8), 748-766 (2004)

18. Wang, G., Sreeram, V., Liu, W.Q.: A new frequency-weighted balanced truncation method and an error bound. IEEE Trans. AC 44(9), 1734-1737 (1999)

19. Houlis, P., Sreeram, V.: A parametrized controller reduction technique via a new frequency weighted model reduction formulation. IEEE Trans. AC 54(5), 1087-1093 (2009)

20. Sreeram, V., Agathoklis, P.: Model reduction using balanced realizations with improved low frequency behaviour. SCL 12(1), 33-38 (1989)

21. Zhou, K.: Frequency-weighted $L_{\infty}$ norm and optimal Hankel norm model reduction. IEEE Trans. AC 40(10), 1687-1699 (1995)

22. Iwasaki, T., Hara, S.: Generalized KYP lemma: unified frequency domain inequalities with design applications. IEEE Trans. AC 50(1), 41-59 (2005)

23. Rantzer, A.: On the Kalman-Yakubovich-Popov lemma. SCL 28(1), 7-10 (1996)

24. Zhou, K., Doyle, J.C., Glover, K.: Robust and optimal control. Prentice Hall, Upper Saddle River, NJ (1996)

25. Lancaster, P., Tismenetsky, M. The theory of matrices, 2nd edn. Academic, Orlando (1985)

26. Barrachina, S., Benner, P., Quintana-Ortí, E.: Parallel algorithms for balanced truncation of large-scale unstable systems. In: Proc. 44rd IEEE Conf. Decision Contr. and European Contr. Conf. ECC2005, pp. 2248-2253. Omnipress, Madison, WI (2004). CD Rom

27. Benner, P., Castillo, M., Quintana-Ortí, E.S., Quintana-Ortí, G.: Parallel model reduction of largescale unstable systems. In: Joubert, G.R., Nagel, W.E., Peters, F.J., Walter, W.V. (eds.) Parallel Computing: Software Technology, Algorithms, Architectures \& Applications. Proc. Intl. Conf. ParCo2003, Dresden, Germany, Advances in Parallel Computing, vol. 13, pp. 251-258. Elsevier B.V. (North-Holland) (2004)

28. Benner, P., Saak, J., Uddin, M.M.: Balancing based model reduction for structured index-2 unstable descriptor systems with application to flow control. Numer. Algebra. Control Optim. 6(1), 1-20 (2016). https://doi.org/10.3934/naco.2016.6.1

29. Zhou, K., Salomon, G., Wu, E.: Balanced realization and model reduction for unstable systems. Int. Rob. Cont. 9(3), 183-198 (1999)

Publisher's note Springer Nature remains neutral with regard to jurisdictional claims in published maps and institutional affiliations. 


\section{Affiliations}

\section{Peter Benner ${ }^{1,2}$ (D) $\cdot \mathrm{Xin} \mathrm{Du}^{3} \cdot$ Guanghong Yang ${ }^{4} \cdot$ Dan Ye $^{4}$}

Xin Du

duxin@shu.edu.cn

Guanghong Yang

yangguanghong@ise.neu.edu.cn

Dan Ye

yedan@ise.neu.edu.cn

1 Max Planck Institute for Dynamics of Complex Technical Systems, Sandtorstr. 1, 39106 Magdeburg, Germany

2 Faculty of Mathematics, Otto von Guericke University, Universitätsplatz 2, 39106 Magdeburg, Germany

3 School of Mechatronic Engineering and Automation, Shanghai University, Shanghai, 200072, , People's Republic of China

4 College of Information Science and Engineering, Northeastern University, Shenyang, Liaoning, 110004, People's Republic of China 